\title{
\begin{tabular}{l|l} 
POLITIQUES \& & Politiques et management public
\end{tabular}

\section{Peut-on discipliner le pouvoir ? Étude sur le contrôle de la rationalité managériale par le juge dans trois innovations législatives françaises}

Can power be disciplined? A study on judges' control of managerial rationality in three French legislative innovations

\section{Patrick Gibert et Pierre-Éric Verrier}

\section{OpenEdition \\ Journals}

Édition électronique

URL : http://journals.openedition.org/pmp/10177

ISSN : 2119-4831

\section{Éditeur}

Institut de Management Public (IDPM)

\section{Édition imprimée}

Date de publication : 30 décembre 2016

ISBN : : 978-2-7430-2327-0

ISSN : 0758-1726

Référence électronique

Patrick Gibert et Pierre-Éric Verrier, « Peut-on discipliner le pouvoir? Étude sur le contrôle de la rationalité managériale par le juge dans trois innovations législatives françaises », Politiques et management public [En ligne], Vol 33/3-4 | 2016, mis en ligne le 04 janvier 2021, consulté le 26 janvier 2021. URL : http://journals.openedition.org/pmp/10177 


\title{
Peut-on discipliner ${ }^{1}$ le pouvoir ? Étude sur le contrôle de la rationalité managériale par le juge dans trois innovations législatives françaises
}

\author{
$>$ Patrick Gibert $^{a^{*}}$ et Pierre-Éric Verrier ${ }^{b}$
}

a Université de Paris Ouest, 200 avenue de la République, 92000 Nanterre

${ }^{b}$ Axes management, 84 rue Amelot, 75011 Paris

\section{Résumé}

Il y a un paradoxe à constater que des normes se proposant d'introduire une forme de rationalité managériale dans la gestion publique sont portées par des textes juridiques de niveau très élevé (en France Loi et loi organique), alors que les analystes mettent fréquemment en relief les contradictions entre cette rationalité managériale et la logique politique, corollaire de la lutte pour le pouvoir et de sa conservation.

L'émergence de la rationalité managériale affaiblirait-elle la domination de la logique politique, lui disputant même sa primauté? Peut-on réellement discipliner le politique en donnant force obligatoire aux bonnes pratiques de management contenues dans la Loi?

Cet article évalue la question de la discipline du politique en étudiant la mise en œuvre de trois textes aux ambitions managériales claires : la loi organique relative aux lois de finances (LOLF) de 2001 instituant l'obligation, pour les pouvoirs publics, de présenter des projets et des rapports annuels de performance à l'appui de chacun de leurs programmes, la loi organique du 15 avril 2009 rendant obligatoires les études d'impact pour les projets de Loi déposés par le Gouvernement, et enfin l'ordonnance du 23 juillet 2015 imposant à l'État et aux collectivités territoriales l'évaluation du mode de réalisation choisi et les études préalables associées aux marchés de partenariat.

Les jurisprudences constitutionnelle et administrative, relatives aux trois textes étudiés, révèlent une prudence extrême du juge pour faire respecter l'ambition du législateur de discipliner le politique du point de vue managérial. L'article suggère plusieurs explications à ce comportement qui, pour n'être pas nouveau, confirme une jurisprudence traditionnelle visant plus à censurer l'irrationalité manifeste qu'à imposer une forme précise de rationalité.

(c) 2016 IDMP/Lavoisier SAS. Tous droits réservés

Mots clés : gouvernance, LOLF, études d'impact, marchés de partenariat, discipline managériale, contrôle, rationalité managériale, analyse d'impact de la réglementation.

1 Par " discipline », nous entendons « une règle de conduite, et par extension, l'obéissance à cette règle » (Dictionnaire Le Robert).

*Auteur correspondant : patrick.c.gibert@orange.fr

doi :10.3166/pmp.33.3-4.165-196 @ 2016 IDMP/Lavoisier SAS. Tous droits réservés 


\section{Abstract}

Can power be disciplined? A study on judges' control of managerial rationality in three French legislative innovations. New norms proposing to introduce some form of managerial rationality in public management are now at the top of the hierarchy (in France, Law and Organic Law). However, analysts frequently point out contradictions between this managerial rationality and the political logic that is a corollary of the struggle for the seizure and preservation of power.

Would the emergence of managerial rationality weaken the dominance of political logic to the point of challenging its supremacy? Can we really discipline political power by making good management practices binding by Law?

This article investigates the question of disciplining the political power by studying the implementation of three texts with clear managerial ambitions : (i) the Organic Law on Finance Laws (LOLF) of 2001 which establishes the requirement for public authorities to submit project proposals and annual performance reports for each of their programs, (ii) the Organic Act of April 15, 2009 which makes impact evaluation mandatory for government bills, (iii) the Ordinance of July 23, 2015 which obliges the State and regional/local authorities to appraise the delivery method and conduct preliminary studies for partnership contracts. The constitutional and administrative jurisprudence related to the three texts studied shows that the judge exhibits extreme prudence in enforcing the ambition of the legislator to impose managerial discipline on political power. The article suggests several reasons for this behavior which characterizes a traditional jurisprudence aiming to censor blatant irrationality rather than impose a precise form of rationality. @ 2016 IDMP/Lavoisier SAS. Tous droits réservés

Keywords : Governance, LOLF, Impact Evaluation, Public-Private Partnerships, Managerial Discipline, Control,

Managerial Rationality, Regulatory Impact Analysis.

\section{Introduction}

Quelle que soit la définition que l'on donne du management public et la finalité qu'on lui assigne, la confrontation des logiques managériale et politique constitue en elle-même un objet scientifique (Gibert, 2015). Les caractéristiques du management public découlent, pour une bonne part, de la confrontation, dans le champ des affaires publiques, d'une logique politique caractérisée notamment par le flou et l'ambiguïté de nombreux objectifs (Chun et Rainey, 2005), l'importance accordée aux symboles, l'inconstance des politiques publiques due à la forte volatilité des priorités, la force du court terme qui s'impose aux gouvernants... et celle d'une rationalité gestionnaire structurée en principe par une stratégie, par des priorités et les renoncements qui vont avec, par les anticipations qui les sous-tendent, par la cohérence entre les moyens utilisés et les objectifs poursuivis, par un minimum de stabilité des objectifs et des priorités... (Crête, 2014)

À suivre cette vision, les « progrès » du management public se feraient-ils nécessairement aux dépens de la logique politique? Quels seraient alors les points d'équilibre pour faire progresser la rationalité managériale tout en ménageant à la logique politique son inévitable primauté? Il y a derrière la question de l'émergence du management public et de sa mise en œuvre effective, le problème de sa distance avec le politique. 
La première façon d'œuvrer pour l'extension de la rationalité managériale est de tenir les gouvernants à distance de l'action publique quotidienne en créant des agences préservées de la pure logique politique par le mode de désignation de leurs dirigeants et la longueur de leur mandat, ainsi que par le recrutement de managers à leur tête. Ce fut une voie privilégiée au Royaume-Uni en particulier par les tenants du New Public Management (N.P.M).

La seconde façon de procéder est de discipliner le politique en lui imposant des pratiques et des standards managériaux. On veut dire par là le contraindre, par la loi, à adopter des règles de « bonne gestion ». Il s'agit ici de poser la question de la force obligatoire des règles de « bonne gestion » publique et de leur censure par le juge. La managérialisation du droit ou la juridisation du management (Chevalier, 1993 ; Bezes, 2005, 2008) qui a suscité, dans la période précédente, de nombreuses contributions de la part des juristes, des sociologues du droit ou des politiques publiques (Duran, 1993 et 2009) n'est effective que si les règles en question se situent à un niveau élevé dans la hiérarchie des normes juridiques et que le juge fait des outils, des méthodes, des standards du management, un sujet de droit ${ }^{2}$.

Quelle portée, le juge donne-t-il donc aux pratiques et aux outils de «bonne gestion publique » inscrits dans le droit positif ? Le juge prend-il au mot le législateur lorsque celuici exprime vouloir discipliner- managérialement parlant- le politique?

Le contrôle de la décision politique s'est construit en France, on le sait, autour d'une doctrine forte de la séparation des pouvoirs (Jan, 2012; Troper, 1973, 2014). Mais paradoxalement, l'autonomie des pouvoirs exécutif et législatif s'est adossée au dualisme juridictionnel qui institue un juge administratif aux côtés du juge de droit commun pour connaître des litiges nés de l'action publique. Il s'agit ici pour le juge administratif non pas de censurer la loi dans ses finalités et son exigence de conformité à la Constitution, mais de s'assurer de sa correcte application à l'action quotidienne de l'administration (Kelsen, 1996).

Les territoires respectifs étant tracés entre les fondements du politique et le contrôle juridictionnel de l'action publique, il reste à constater que les faits soulevés devant la juridiction administrative ont, encore récemment et à de multiples occasions, interrogé la rationalité managériale, fût-ce parfois de biais, et souvent en la frôlant. Mais ce processus de managérialisation du droit renforce l'effectivité de la règle et la capacité du juge à s'approprier le thème de la bonne gestion ${ }^{3}$ (Caillosse, 2003).

De fait, l'émergence dans la jurisprudence administrative de la proportionnalité de la décision publique avec ses impacts prévisibles, et notamment l'examen du bilan coûts/ avantages, a tôt posé la question de la capillarité du contrôle de légalité interne et du solde

\footnotetext{
${ }^{2}$ L'un des juges assesseurs à la Cour suprême des États-Unis, emporté par son lyrisme, posait déjà en 1922 la question de l'effectivité de la règle de bonne gestion figurant dans la loi : « Les obligations juridiques qui existent, mais qui ne peuvent pas être appliquées, sont des fantômes qui passent dans la loi, et qui ne peuvent être étreints. ", Oliver Wendel Holmes Jr., aff. The Western Maid, 257, US 419, 1922. [Problème de l'immunité du pouvoir fédéral soulevée à propos d'un navire marchand affrété par la Marine américaine et victime d'une collision.]

${ }^{3}$ Jacques Caillosse, 2003 : « En soumettant, globalement, l'exercice de la fonction administrative, ou si l'on préfère de l'action publique, à des préoccupations de type managérial; en obligeant peu à peu et tant bien que mal des acteurs publics surtout formés à évaluer leur « production » du point de vue de sa régularité juridique, à une interrogation persistante sur la qualité de leurs prestations (.../...) les politiques de réforme de l'État, par leur inspiration managériale rendent de plus en plus artificiels et problématiques la plupart des grands découpages de la réalité par lesquels procède la pensée juridique pour donner à voir un monde ordonné, dans le but de le conformer à ses images. », p. 10.
} 
systémique, fondement de la rationalité managériale : le bilan coûts/avantages, apparu dans les décisions du Conseil d'État en 1971, semble en apparence poser la question de l'évaluation des inputs et des outputs, central pour tout processus managérial, et fondement reconnu des bonnes pratiques de gestion. La décision connue sous le nom de Ville nouvelle Est ${ }^{4}$, par son audace, avait suscité de larges espoirs dans la sphère des praticiens du management public. Le droit positif allait se mettre en phase avec les exigences instrumentales de l'évaluation au moment même où le management public commençait à émerger en France.

Malgré les limites que le juge fixa lui-même à son propre contrôle, notamment en interdisant la comparaison avec les projets alternatifs portés par les requérants ${ }^{5}$, la décision Ville nouvelle Est a vite montré son extraordinaire faculté d'adaptation : de récentes décisions illustrent la capacité du juge administratif à transposer la théorie du bilan coût/avantages dans des domaines où elle n'était pas prévue à l'origine, tel que le principe de précaution contribuant désormais à la notion d'utilité publique ${ }^{6}$ (Godard, 2000). Certains tempéraments inquiets avaient un moment redouté un basculement managérial du juge administratif et la dénaturation de la notion d'intérêt général qui constitue le fondement traditionnel de l'action publique ${ }^{7}$. Mais, on semble en réalité encore bien loin, avec le bilan coûts/avantages, d'une véritable « acculturation managériale » entreprise par le juge administratif (Verrier, 1985).

L'erreur manifeste d'appréciation, quant à elle, constitue une discipline du politique et un contrôle de l'action publique ${ }^{8}$. Du moins, l'a-t-elle revendiquée. Sans doute, cette appréciation est-elle largement conditionnée par le fait que l'erreur manifeste d'appréciation est apparue dans le champ du contrôle restreint du juge, précisément là où la décision politique se fait discrétionnaire, libre de ses appréciations.

Mais, le juge administratif, soucieux de ne pas s'immiscer sur le terrain de la décision, a limité ici considérablement son contrôle en s'interdisant l'exploration de champs entiers de l'action publique. Ainsi, le choix entre deux tracés de lignes de chemin de fer n'est pas susceptible d'être porté devant la juridiction administrative ${ }^{9}$. De même, le choix entre deux modes de gestion d'un service public ne peut faire l'objet d'un recours ${ }^{10}$.

Alors, lorsque la loi exige la mise en place d'un indicateur de performance par l'État, cette obligation est-elle prise au mot par le juge ? À l'inverse, la managérialisation du droit ne reposerait-elle que sur une «soft law » d'interprétation très large et peu contraignante, alors même que la production juridique des nouvelles normes managériales emprunte des voies de plus en plus solennelles. Ce fut d'abord la circulaire, puis le décret, la loi et enfin

\footnotetext{
${ }^{4} \mathrm{CE}, 28$ mai 1971, req. 78825, Ministre de l'équipement et du logement c/Fédération de défense des personnes concernées par le projet actuellement dénommé «Ville nouvelle Est »- Rec. Lebon p. 409

${ }^{5}$ CE, sect., 7 octobre 1977, Syndicat des paludiers et al., req. 99986, Rec. p. 380

${ }^{6} \mathrm{CE}, 12$ avril 2013, Association coordination interrégionale stop $T H T$, réq. № 342409

${ }^{7}$ Caillosse J., 1989 : «Avec l'intérêt général, on tient probablement le lieu principal par où le champ juridique s'ouvre aux influences extérieures et surtout à celles de l'économie et du marché. Une fonction de l'intérêt général dont il est difficile de douter depuis sa codification par le Conseil d'État, dans ce qu'on appelle la jurisprudence du «bilan » (.......). Comment reprocher au juge une indifférence aux impératifs de plus grande rigueur administrative : les méthodes $R C B$ ont trouvé dans la pratique du bilan coût-avantages une manière d'équivalent jurisprudentiel! », p. 177.

${ }^{8}$ CE, ass., 21 juillet 1970, Lambert, req. 76230, 76231, 76235

${ }^{9} \mathrm{CE}, 3$ décembre 1990, Ville d'Amiens, Rec. p. 344

${ }^{10} \mathrm{CE}, 10$ janvier 1992, Association des usagers de l'eau de Peyreleau, Rec. p. 13
} 
la loi organique ${ }^{11}$. On assiste en effet à une véritable montée de ces innovations dans la hiérarchie des normes juridiques, ce qui devrait en toute logique conduire à leur plus grande effectivité en droit positif.

Notre étude est fondée sur l'examen des disciplines instaurées par trois textes promulgués depuis le début de ce siècle : la loi organique relative aux lois de finance (LOLF), la loi organique instaurant l'obligation d'une étude d'impact à l'appui de (presque) tous les projets de loi déposés par le Gouvernement, et l'ordonnance créant les marchés de partenariat (voir encadré 1). Dans la première partie de notre article nous présenterons les dispositifs qui semblent, au sein de ces textes, relever de la rationalité managériale avant d'analyser ce que leurs origines, les insatisfactions du monde politico-administratif quant à leur mise en œuvre ainsi que les propositions de réformes qui en sont avancées peuvent révéler comme ambiguïtés, multiplicité voire contradictions d'objectifs et incertitude sur la nature réelle de la discipline demandée (Partie 1). Dans un second temps, nous illustrerons à partir de la jurisprudence constitutionnelle et/ou administrative, l'idée selon laquelle la position prudente du juge renforce le caractère managérial très relatif de la discipline instaurée (Partie 2).

\section{Encadré 1 : Indications méthodologiques}

Choix de trois obligations instaurées par le législateur organique ou le législateur tendant à faire expliciter par le pouvoir exécutif (niveau national) et les exécutifs des collectivités territoriales les raisons des choix qu'ils proposent au pouvoir législatif ou aux assemblées délibérantes (en ce qui concerne les collectivités territoriales)

Pour l'obligation en matière d'explicitation d'objectifs et d'indicateurs pour chaque programme ainsi qu'en matière de comptabilité et d'analyse des coûts (Projets annuels de performance annexés aux projets de lois de finances initiales) :

- Étude de la jurisprudence du Conseil constitutionnel

- utilisation des recherches de Benzérafa et al. (2011), Benzérafa et Gibert (2015), Benzérafa, Garcin et Gibert (2016), en ce qui concerne la qualité des liens entre stratégie, objectifs et indicateurs

Pour les études d'impact devant accompagner le dépôt d'un projet de loi :

- Étude de la jurisprudence du conseil constitutionnel,

- Étude de la position du conseil d'État dans son rôle de conseiller du gouvernement.

- Analyse du débat en cours sur les modifications apportées à la procédure (AN 2014, CE 2016).

- Analyse qualitative de trois études d'impact.

Pour l'étude préalable aux contrats puis marchés de partenariat :

- Étude de cas sur deux exemples

${ }^{11}$ En novembre 2003, à quelques semaines de la mise en œuvre de la LOLF, un haut fonctionnaire de l'administration des finances se réjouissait pourtant de pouvoir « réformer le budget de la France par Power Point »...Frank Mordacq, Colloque PMP « Une génération de réformes en management public : et après? », à l'ENA - Strasbourg, 24-25 novembre 2003. 


\section{Des dispositions semblant concourir à la rationalité managériale mais dont l'origine et la mise en œuvre révèlent une nature plus complexe}

\subsection{Les indices d'une rationalisation managériale dans les trois textes}

Forts sans doute d'une distanciation supérieure, ce sont les non gestionnaires qui s'efforcent de cerner la rationalité managériale. Dans une approche inspirée par Foucault (1975), Le Texier (2011) l'assimile à la « gouvernementalité » managériale en la caractérisant par quatre principes cardinaux : l'efficacité à travers la mise en exergue de la production d'effets, l'organisation structurée par la recherche des résultats attendus, le contrôle sous forme d' «influence », prescription et injonction centrées sous la soumission à des normes objectivées, et finalement le savoir remontant par la collecte d'informations et son traitement et s'imposant par la standardisation, par la planification et par la formation technique des individus.

Dans une approche plus instrumentaliste, Vigour (2006) résumant la pensée de Philippe Bezes (2002) pose que « la mise en cuvre d'une rationalité de type managérial se caractérise par l'attention aux outils de gestion, à la mesure des performances (grâce à la création d'indicateurs), à l'évaluation de l'action (par la mise en place de contrôles ex post), à l'efficience, à la maîtrise des coûts de l'action publique (impliquant un raisonnement fondé sur le calcul coûts/bénéfices), à la relation à l'usager et à la gestion du personnel » (p 428).

Quel que soit le sentiment qu'un gestionnaire peut avoir sur ces visions de la rationalité managériale et sur le fait qu'elles paraissent mélanger vision ontologique et vision normative du management (Gibert, 2015), on doit les prendre en grande considération car c'est sur elles que se fondent généralement les appréciations des analystes qui mettent en avant une managerialisation de la chose publique. Cependant, s'agissant des affaires publiques, ces présentations de la rationalité managériale ne suffisent pas à la différencier de la rationalité politique ou à l'opposer à celle-ci. Pour ce faire, il convient de rajouter l'analyse de système; analyse de système conçue d'un point de vue essentiellement économique comme cela a pu être reproché à celle qui imprégnait le PPBS (Planning Programming Budgeting System américain, et par décalque la rationalisation des choix budgétaire (RCB ) française, ou bien élargie par la prise en compte des sciences sociales ce qui pour certains (Dror, 1967, Wildavsky, 1968) était la base de l'analyse de politique (dans sa version utilitariste). Cette analyse soulignant le caractère non mécanique mais plutôt interactionniste de la chaîne de causes à effets, la multiplicité et la variété possible des effets non recherchés voire pervers engendrés par toute action publique, leur importance dans les résultats à attendre d'une décision, ainsi que la vision que peuvent en avoir les parties (volontairement ou non) prenantes s'oppose en effet au simplisme d'une vision politique trop centrée sur un effet, au détriment de tous les autres, ou sur un moyen auquel est attaché trop de valeur.

Mettons à présent en exergue dans les lois sur lesquelles notre étude a été centrée les dispositions qui peuvent être interprétées comme visant à renforcer la rationalité managériale des décideurs publics.

- La loi organique relative aux lois de finances (LOLF) de 2001 a été qualifiée de «Constitution financière de l'État», cette appellation, juridiquement fausse, a le mérite de souligner l'importance de la novation qu'elle a introduite dans la présentation, le vote et l'exécution du budget de l'État, instrument essentiel de la vie et de la gestion publiques 
dans toute démocratie. Le budget est désormais voté par mission et selon l'article 7 de la loi : «Une mission comprend un ensemble de programmes concourant à une politique publique définie » et «Un programme regroupe les crédits destinés à mettre en œuvre une action ou un ensemble cohérent d'actions relevant d'un même ministère et auquel sont associés des objectifs précis, définis en fonction de finalités d'intérêt général, ainsi que des résultats attendus et faisant l'objet d'une évaluation ». La LOLF dispose dans son article 51 que les annexes du projet de loi de finance sont accompagnées « du projet annuel de performances de chaque programme précisant : la présentation des actions, des coûts associés, des objectifs poursuivis, des résultats obtenus et attendus pour les années à venir mesurés au moyen d'indicateurs précis dont le choix est justifié ». L'article 54 de la LOLF énonce que parmi les pièces jointes au projet loi de règlement -qui vise à obtenir l'approbation du Parlement pour les comptes d'une année- figurent « Les rapports annuels de performances, faisant connaître, par programme, en mettant en évidence les écarts avec les prévisions des lois de finances de l'année considérée, ainsi qu'avec les réalisations constatées dans la dernière loi de règlement : a) Les objectifs, les résultats attendus et obtenus, les indicateurs et les coûts associés...».

Projets annuels de performance (PAP) et Rapports annuels de performance (RAP) incluent également des données issues de la comptabilité analytique des coûts (CAC). Selon l'article 27 de la LOLF «L'État tient une comptabilité des recettes et des dépenses budgétaires et une comptabilité générale de l'ensemble de ses opérations. En outre, il met en ouvre une comptabilité destinée à analyser les coûts des différentes actions engagées dans le cadre des programmes. Les comptes de l'État doivent être réguliers, sincères et donner une image fidèle de son patrimoine et de sa situation financière ». L'article 51 de la loi organique dispose que les projets annuels de performance incluent « la présentation des actions, des coûts associés »; ceux-ci doivent également être présentés dans les RAP. L'examen des PAP et de RAP montre l'importance de l'enjeu théorique que cette présentation revêt. Si les crédits sont attribués pour des programmes, une présentation indicative en est effectuée par action. Le fait que la structuration des programmes et des actions ait largement été conditionnée par des considérations de structure organisationnelle, bien plus que par une structure par objectifs, implique qu'une action peut consommer des ressources très largement différentes des crédits qui lui sont affectées. Une action peut ainsi disposer de ressources correspondant à des crédits enregistrés pour une autre action, voire pour un autre programme...

- La loi du 15 avril $2009^{12}$ vise à la mise en œuvre de dispositions nouvelles introduites dans la Constitution par la révision de celle-ci opérée en 2008 (voir encadré 2). Comme telle, elle se présente comme un effort de rééquilibrage en faveur du pouvoir législatif, sérieusement amputé par le texte initial de la Constitution de la Ve République. Son article 8 rend obligatoire l'étude d'impact, précédemment pratiquée en matière d'environnement et par ailleurs en vertu de normes de niveaux inférieurs ${ }^{13}$ pour les projets de loi déposés par le Gouvernement. Cet article prévoit que l'étude d'impact doit comprendre « l'évaluation des conséquences économiques, financières, sociales et environnementales, ainsi que des

\footnotetext{
${ }^{12}$ La loi organique n²009-403 du 15 avril 2009 relative à l'application des articles 34-1, 39 et 44 de la Constitution

${ }^{13}$ De façon expérimentale par une circulaire du 21 novembre 1995, de façon généralisée par une circulaire du 26 janvier 1998, dispositif remanié par une troisième circulaire en septembre 2003 (cf. Warsmann, 2009)
} 
coûts et bénéfices financiers attendus des dispositions envisagées pour chaque catégorie d'administrations publiques et de personnes physiques et morales intéressées en indiquant la méthode de calcul retenue ». Cela laisse entendre une obligation pour le pouvoir exécutif d'accompagner tous ses projets d'une véritable évaluation a priori - type d'évaluation qui était au premier rang de la rénovation annoncée de la gestion publique du temps de la Rationalisation des Choix Budgétaires, dont la pratique a perduré dans certains domaines : routes, lignes de chemin de fer à grande vitesse... mais qui, dans l'agenda politique, a cédé la place depuis longtemps à l'évaluation a posteriori.

\section{Encadré 2 : Loi organique $n^{\circ}$ 2009-403 du 15 avril 2009 relative à l'application des articles 34-1, 39 et 44 de la Constitution. Chapitre II : dispositions relatives à la présentation des projets de loi prises en vertu de l'article 39 de la constitution}

\section{Article 8}

Les projets de loi font l'objet d'une étude d'impact. Les documents rendant compte de cette étude d'impact sont joints aux projets de loi dès leur transmission au Conseil d'État. Ils sont déposés sur le bureau de la première assemblée saisie en même temps que les projets de loi auxquels ils se rapportent.

Ces documents définissent les objectifs poursuivis par le projet de loi, recensent les options possibles en dehors de l'intervention de règles de droit nouvelles et exposent les motifs du recours à une nouvelle législation.

Ils exposent avec précision :

.......

- l'évaluation des conséquences économiques, financières, sociales et environnementales, ainsi que des coûts et bénéfices financiers attendus des dispositions envisagées pour chaque catégorie d'administrations publiques et de personnes physiques et morales intéressées, en indiquant la méthode de calcul retenue; - l'évaluation des conséquences des dispositions envisagées sur l'emploi public; - les consultations qui ont été menées avant la saisine du Conseil d'État;

De son côté, l'ordonnance n 2015-899 du 23 juillet 2015, a changé le contrat de partenariat, lui-même régi par plusieurs vagues législatives différentes, issues de majorités politiques elles-mêmes différentes en marché de partenariat et en a donné une définition relativement large : «Un marché de partenariat est un marché public qui permet de confier à un opérateur économique, une mission globale ayant pour objet : $1^{\circ} \mathrm{La}$ construction, la transformation, la rénovation, le démantèlement ou la destruction d'ouvrages, d'équipements ou de biens immatériels nécessaires au service public ou à l'exercice d'une mission d'intérêt général ; $2^{\circ}$ Tout ou partie de leur financement. (.../...) II. - Cette mission globale peut également avoir pour objet : (.../...) la gestion d'une mission de service public ou des prestations de services concourant à l'exercice, par la personne publique, de la mission de service public dont elle est chargée ».

Les marchés de partenariat, aujourd'hui rangés dans la catégorie des marchés publics, aménagent les modalités classiques sur lesquelles reposent la passation et la gestion des 
marchés publics essentiellement sur trois points : leur durée, inhabituellement longue pour des contrats de la commande publique (20 à 30 ans dans la pratique, car les textes ne fixent comme limite de durée que la durée des amortissements et/ou les modalités de leur financement); la dérogation à la règle de l'appel d'offres; l'introduction dans les marchés de partenariat de clauses de paiement différé, interdites par l'article 60-I du décret du 25 mars 2016 relatif aux marchés publics.

Afin de dissiper les craintes exprimées par la doctrine et nombre de praticiens, mais également pour discipliner les décideurs qui souhaiteraient recourir aux marchés de partenariat, les pouvoirs publics ont inscrit, dès l'ordonnance du 17 juin 2004 et plus encore dans l'ordonnance du 23 juillet 2015, des conditions de recours aux marchés de partenariat, et en particulier l'obligation d'une étude préalable justifiant ce recours (voir encadré 3).

\section{Encadré 3 : Ordonnance $n^{\circ}$ 2015-899 du 23 juillet 2015 relative aux marchés publics (extrait)}

«Article 74 - Évaluation et études préalables

La décision de recourir à un marché de partenariat, quel que soit le montant d'investissement, doit être précédée de la réalisation de l'évaluation du mode de réalisation du projet prévue à l'article 40. L'acheteur réalise également une étude de soutenabilité budgétaire qui apprécie notamment les conséquences du contrat sur les finances publiques et la disponibilité des crédits.

Article 75 - Conditions de recours

I. - La procédure de passation d'un marché de partenariat ne peut être engagée que si l'acheteur démontre que, compte tenu des caractéristiques du projet envisagé, des exigences de service public ou de la mission d'intérêt général dont l'acheteur est chargé, ou des insuffisances et difficultés observées dans la réalisation de projets comparables, le recours à un tel contrat présente un bilan plus favorable, notamment sur le plan financier, que celui des autres modes de réalisation du projet. Le critère du paiement différé ne saurait à lui seul constituer un avantage. (.../...) II. - Les acheteurs ne peuvent recourir au marché de partenariat que si la valeur de ce marché est supérieure à un seuil fixé par voie réglementaire en fonction de la nature et de l'objet du contrat, des capacités techniques et financières de l'acheteur et de l'intensité du risque encouru. »

L'examen des trois textes étudiés imposant une discipline, ainsi que des documents conçus pour leur mise en œuvre, révèle des emprunts significatifs, explicites ou implicites suivant les cas au langage managérial public.

Le premier trait commun aux trois disciplines instituées est la référence à la notion d'évaluation. Pour l'étude d'impact le projet de loi présenté par le gouvernement prévoyait initialement « un ou plusieurs documents qui rendent compte des travaux d'évaluation préalable réalisés », c'est par un amendement proposé en commission que ces travaux d'évaluation préalable sont devenus « étude d'impact » au motif que cela avait été le terme utilisé dans les dispositifs antérieurs (AN 2009). Ce lien avec l'évaluation est confirmé dans un texte d'application « La loi organique définit le contenu de l'étude d'impact. Cette étude 
n'est pas assimilable à un exposé des motifs enrichi, mais constitue un outil d'évaluation et d'aide à la décision $\gg^{14}$. Dans le contrat devenu marché de partenariat (cf. encadré 3 ), c'est d'évaluation du mode de réalisation du projet dont il s'agit. En ce qui concerne la LOLF, s'il n'est question que de « présentation » des résultats attendus » à l'article 51 l'expression d'évaluation apparaît pour les mesures proposées au sein du projet de lois de finances ${ }^{15}$, donc pour les changements envisagés ayant un impact sur les charges ou ressources de l'année à venir. Les trois dispositifs étudiés se rattachent donc au principe d'évaluation $e x$ ante à une époque où l'évaluation ex post connaît diverses vicissitudes. ${ }^{16}$

Pour deux des trois instruments la logique affichée est une logique d'optimisation. Optimisation en creux pour l'étude d'impact dont les documents qui la constituent «définissent les objectifs poursuivis par le projet de loi, recensent les options possibles en dehors de l'intervention de règles de droit nouvelles et exposent les motifs du recours à une nouvelle législation. » (Article 8 de la loi organique), optimisation explicitement réclamée comme conclusion des études préalables des marchés de partenariat puisqu'il convient de faire apparaître que "le recours à un tel contrat présente un bilan plus favorable, notamment sur le plan financier, que celui des autres modes de réalisation du projet. » (Article 75 précité). Les PAP, quant à eux, ne s'inscrivent pas dans une perspective d'optimisation, mais dans celle d'une performance par nature éclatée puisqu'estimée au moyen d'indicateurs généralement non agrégeables et non agrégés. La différence est de taille mais doit être relativisée compte tenu du fait que dans les cas de référence implicite ou explicite à l'optimisation, celle-ci se fait sur une pluralité de critères dont l'identité non plus que la logique d'articulation ou de pondération ne sont précisés.

La référence explicite à l'analyse de système n'a été faite dans aucun des dispositifs étudiés, mais les idées principales de cette analyse se retrouvent dans les textes en question. Ainsi, pour l'étude d'impact le guide de légistique pose que «Il s'agit d'une méthode destinée à éclairer les choix possibles, en apportant au Gouvernement et au Parlement les éléments d'appréciation pertinents : nature des difficultés à résoudre, avantages et inconvénients des options possibles en fonction de l'objectif poursuivi, évaluation détaillée des conséquences qui peuvent être raisonnablement attendues de la réforme pour chacune des catégories de personnes concernées comme pour les administrations elles-mêmes...». Dans le cas de l'étude préalable au marché de partenariat, le centrage sur un projet d'équipement dont la raison d'être n'a pas à être justifiée contrairement au mode de réalisation choisi, la référence implicite à l'analyse de système est moins visible mais non absente dès lors que l'évaluation comparative doit mettre l'accent non seulement sur les coûts mais aussi sur une performance qui peut être multiforme, sur le partage des risques entre la personne publique et son co-contractant privé,

\footnotetext{
${ }^{14}$ Premier Ministre, Circulaire du 15 avril 2009 relative à la mise en œuvre de la révision constitutionnelle (procédure législative)

${ }^{15}$ Article 55 de la LOLF «Chacune des dispositions d'un projet de loi de finances affectant les ressources ou les charges de l'État fait l'objet d'une évaluation chiffrée de son incidence au titre de l'année considérée et, le cas échéant, des années suivantes » Cette obligation concerne «Tous les articles fiscaux, qu'ils figurent en première partie ou en seconde partie de loi de finances; Les dispositions relatives aux autres ressources de l'État inscrites en première partie de la loi de finances, dès lors qu'elles affectent l'équilibre budgétaire : Les dispositions de la seconde partie qui relèvent du domaine facultatif et partagé, énumérées au $7^{\circ}$ du II de l'article 34 de la LOLF » (guide de légistique).

${ }^{16}$ Suppression de fait du dispositif interministériel et du Conseil national de l'évaluation instaurés par le Gouvernement Jospin.
} 
ainsi que sur les préoccupations (environnementales, sociales et de développement durable ${ }^{17}$ ). L'absence de référence à l'analyse de système dans le cas des PAP n'est pas étonnante dans la mesure où le recours à cette analyse de système se justifie beaucoup plus dans des logiques de prise de décision et de mise en exergue intertemporelle ou transtemporelle de ses effets que dans une logique de redevabilité périodique d'une entité dont les résultats sont affectés par les conséquences de nombreuses décisions prises dans un passé plus ou moins proche.

\subsection{La concurrence entre rationalisation managériale et d'autres paradigmes assumés}

Le vocabulaire utilisé dans les trois « lois » ou leurs documents d'application ne se réfère que très partiellement au vocabulaire managérial dominant : ainsi, pour l'étude d'impact il n'est pas question de «théorie d'action» ou de «théorie du changement social» ou de « modélisation » pour désigner les chaînes supposées de relations de cause à effet entre moyens utilisés et résultats (de tous niveaux) attendus. De la même façon, les notions (assez constructivistes) intégrées dans la Balanced scorecard (Kaplan et Norton, 2001) par l'intermédiaire de la carte stratégique qui ordonnent, et différencient les objectifs et les indicateurs de différents niveaux ne sont nulle part évoquées ${ }^{18}$, ni mis en œuvre dans les projets annuels de performance (Benzérafa et Gibert, 2015).

Le principe de contextualisation paraît éclipsé par un fort isomorphisme coercitif. Pour l'étude d'impact aucune distinction n'est faite à l'intérieur des projets de loi redevables d'une étude. Alors qu'une typologie multidimensionnelle pourrait être invoquée : loi à champs d'action large $v s$ loi à dispositif très focalisé, loi bouleversant la politique dans un domaine déterminé vs loi amendant marginalement une politique existante, différents domaines concernés : culture, économie, justice, libertés publiques..., différenciation suivant les instruments de gouvernement utilisés : interdiction, réglementation, subventions... Cette indifférence au contexte, au périmètre concerné, aux outils mobilisés etc. apparaît de la même façon quant aux prescriptions de la LOLF en matière de présentation des programmes. Quel que soit le domaine d'action des programmes, quelle que soit leur nature (programme de «politique publique » (c'est-à-dire final) ou de « soutien »... les obligations sont les mêmes ${ }^{19}$, mais aussi dans l'ordonnance relative aux contrats-marchés de partenariat. On pourrait penser que cette adaptation à chaque cas serait traitée dans les textes d'application mais ce n'est pas le cas. Paradoxalement, pour l'étude d'impact c'est le conseil constitutionnel qui a fait preuve de relativisme dans l'une des deux réserves interprétatives qu'il a émises lorsque le texte de la loi organique lui fut soumis (cf. infra).

Les trois instruments de discipline étudiés ne peuvent être rattachés à la seule rationalité managériale, sauf à étendre à l'extrême le sens de celle-ci. Le texte de la LOLF a pu consti-

\footnotetext{
${ }^{17}$ Mission d'appui à la réalisation des contrats de partenariat; guide méthodologique, 2009

${ }^{18}$ À leur place, seulement au niveau des documents d'application de la loi organique et sans référence explicite à la théorie des parties prenantes une différenciation fondée sur le destinataire (usager, citoyen, contribuable) et une correspondance bi univoque établie avec la qualité de service, les effets socio-économiques et l'efficience.

${ }^{19}$ À ceci près que l'isomorphisme est renforcé pour les programmes de soutien (gestion du personnel, gestion financière et comptable...) pour lesquels on doit retrouver des indicateurs communs d'un programme à un autre.
} 
tuer un objet frontière ${ }^{20}$ pour d'importants groupes sociaux (Social worlds) poursuivant des objectifs différents. Le premier d'entre eux était celui des parlementaires désireux de regagner une partie du pouvoir budgétaire qui avait été rogné par l'ordonnance de 1959 et intéressés par une amélioration de l'accountability gouvernementale à leur égard. Le gouvernement de l'époque soucieux d'afficher une opération de modernisation de la gestion publique au champ large qui faisait défaut depuis la mise en sommeil et la fin de l'opération renouveau du service public constituait le deuxième groupe social. Un autre était le monde, principalement académique, de spécialistes en finances publiques désireux de dynamiser une matière peu évolutive, en particulier du fait de l'échec des nombreuses tentatives de réformes avortées de l'ordonnance organique de 1959. Un autre univers composite était celui de fonctionnaires et d'académiques demandeurs d'une modernisation tendant à une meilleure finalisation de l'action publique. On trouvait encore l'univers des fonctionnaires exerçant ou ayant exercé dans l'administration territoriale de l'État et demandeurs d'une plus forte déconcentration au profit des services la composant et de leurs responsables. Un autre monde était celui des tenants de l'isomorphisme mimétique, de l'idée selon laquelle « l'État doit être géré comme une entreprise » des adeptes du «New public management». Il n'est pas étonnant dès lors que la mise en œuvre de la LOLF, objet frontière, ait entraîné dans sa partie PAP comme pour le reste tant de déceptions parmi des alliés aux motivations si diverses.

L'obligation d'effectuer une étude d'impact pour la grande majorité des projets de lois se rattache suivant le temps et les documents à divers paradigmes, celui du rééquilibrage entre pouvoir exécutif et pouvoir législatif, (rapport Balladur 2007), problème de gouvernance par excellence, celui des processus de la confection des normes, paradigme de la légistique qui met l'accent sur les différentes activités et sur la chronologie à respecter pour arriver à une «bonne rédaction » des textes (guide de légistique, fiche 1.1.1), celui de la simplification administrative (AN 2014) (CE 2016) qui part du constat de la multiplication, de la complexification des normes et des coûts qu'elles engendrent pour les administrés et plus particulièrement pour les entreprises ${ }^{21}$. L'obligation de l'étude d'impact peut être aussi rattachée à la lutte contre la « loi bavarde », paradigme cher aux juges administratif et constitutionnel s'élevant contre le développement de passages sans portée normative dans

\footnotetext{
${ }^{20}$ L'objet frontière est « un concept analytique de ces objets scientifiques qui d'une part sont présents dans plusieurs mondes sociaux s'entrecoupant (...) et qui satisfont les besoins d'information de chacun d'entre eux. Les objets frontière sont des objets qui sont suffisamment plastiques pour s'adapter aux besoins locaux et aux contraintes des parties les employant mais cependant assez robustes pour maintenir une identité commune d'un lieu à un autre. Ils sont faiblement structurés dans leur usage commun et deviennent fortement structurés dans l'usage fait dans un lieu particulier. Ces objets peuvent être abstraits ou concrets. Ils ont différents sens dans différents mondes sociaux mais leur structure est suffisamment commune à plus d'un monde pour les rendre reconnaissables, en faire un moyen de traduction. » (Star et Griesemer, 1989)

${ }^{21}$ Le mouvement pour la simplification s'appuyant de plus en plus sur l'idée selon laquelle la « qualité » de la réglementation est un atout ou un désavantage concurrentiel dans la lutte des divers États pour retenir ou attirer les activités économiques.
} 
les textes législatifs ${ }^{22}$. L'idée de l'étude d'impact relève également de la montée en puissance de la redevabilité des gouvernants à l'égard des citoyens ou des différentes parties prenantes à l'application d'une norme, voire de la démocratie participative quand les intéressés sont conviés à participer au processus de l'étude ${ }^{23}$. L'étude d'impact peut donc, elle aussi, être considérée comme un objet frontière dont les alliés ont des motivations qui sont loin de se ramener à la seule rationalité managériale. La question de la compatibilité entre ces différentes attentes, et en particulier avec celle d'une meilleure rationalité managériale, est bien évidemment cruciale quant à l'appréciation de ce que l'on entend par « qualité » des études.

La filiation de l'étude préalable pour les marchés de partenariat paraît plus simple dans la mesure où il s'agit de légitimer le recours à un instrument dérogatoire au droit commun. La supériorité désormais affichée de la rationalité managériale ne supprime cependant pas les difficultés engendrées par l'appréciation multicritère des avantages et des coûts du marché à un stade précoce du projet et aux traductions difficilement objectivables des notions de complexité et d'urgence.

Pour essayer de préciser la portée réelle des disciplines et des contraintes instaurées, il est utile de se référer aux diagnostics énoncés quant aux causes des imperfections constatées par les observateurs ainsi qu'aux idées d'amélioration que ceux-ci ont avancées.

Pour les PAP, le diagnostic avancé ${ }^{4}$ a eu trait à un savoir insuffisant en particulier dans l'explicitation d'une stratégie mettant en perspective les objectifs, dans le choix entre les différentes catégories d'objectifs (de qualité de service, d'effets socio-économiques et d'efficience), dans la définition complète des indicateurs, en résumé dans une technique de finalisation et de la métrique qu'on doit lui associer pour cerner la performance (Benzérafa et Gibert, 2015).

Généralement, la qualité des études d'impact réalisées après la mise en œuvre de la loi organique de 2009 a fait l'objet de critiques nombreuses d'origines variées et parfois très sévères. Ces critiques émanent des parlementaires qui soulignent parmi les mauvaises conditions de la préparation de la loi « l'évaluation insuffisante - voire inexistante - de son impact social, économique, environnemental... (rapport AN 2014), de la haute administration «les études d'impact ressemblent davantage, à l'heure actuelle, à un exposé des motifs enrichi qu'au modèle de l'Organisation de coopération et de développement économique (OCDE),

\footnotetext{
22 "Le premier élément de la dégradation de la norme réside dans le développement des textes d'affichages, un droit mou, un droit flou, un droit à « l'état gazeux » » (Rapport public annuel du Conseil d'État 1991). La loi est « bavarde, précaire, banalisée » fruit d'une gesticulation législative des pouvoirs publics; Renaud Denoix de Saint-Marc (alors vice-président du Conseil d'État), 21 janvier 2001, entretien au Journal du Dimanche. «La loi n'est pas faite pour affirmer des évidences, émettre des vœux ou dessiner l'état idéal du monde (en espérant sans doute le transformer par la seule grâce du verbe législatif?). La loi ne doit pas être un rite incantatoire. Elle est faite pour fixer des obligations et ouvrir des droits. En allant au-delà, elle se discrédite », Pierre Mazeaud (alors président du Conseil Constitutionnel) 2005. Tout ce mouvement rejette au dehors du contenu normal de la loi les aspects symboliques, la quête de sens chère à l'approche cognitive des politiques publiques.

${ }^{23}$ Assez marginalement dans le système français actuel ou chacun peut adresser ses remarques sur l'étude à l'Assemblée qui a reçu en premier le dossier législatif du Gouvernement, de façon plus significative dans le système de la commission européenne ou les parties prenantes doivent être consultées le plus en amont possible.

${ }^{24}$ En particulier par le Comité interministériel d'audit des programmes (CIAP), composé de membres des corps de contrôle ainsi que par la Cour des comptes.
} 
pour laquelle l'étude d'impact sert à ajuster le dispositif qui sera retenu » (Serge Lasvignes, Secrétaire général du gouvernement in ibidem), de membres de l'exécutif même, Jean-Marie Le Guen ${ }^{25}$ expose ainsi que les études d'impact « apparaissent aujourd'hui comme un exercice imposé et prennent parfois la forme d'un "exposé des motifs bis" (ibidem), Thierry Mandon ${ }^{26}$ regrette que l'expertise qui existe dans la société ne soit presque jamais utilisée dans la phase d'élaboration des textes et concède à propos de la loi El Khomry que l'impact social de son article 2 qui fait l'objet de deux lignes aurait à l'évidence mérité des développements beaucoup plus substantiels ${ }^{27}$. Les remarques effectuées par le Conseil d'État méritent une place particulière puisque celui-ci doit donner son avis sur tous les projets de loi avant soumission des dits projets au parlement. Si ces avis sont en principe secrets ${ }^{28}$, les rapports annuels du Conseil donnent un aperçu de leur contenu et en particulier depuis 2010 de l'appréciation des études d'impact qui y figurent. Les appréciations un peu détaillées sont souvent moins sévères que la formulation du Vice-président du Conseil qui relie les défauts des dites études à «notre goût des villages Potemkine » (Conseil d'État, 2016), goût auquel il convie à renoncer, elles sont cependant préoccupantes. Dans le rapport public 2013, à côté de la reconnaissance de progrès, le Conseil d'État souligne la nécessité d'améliorations sensibles, en particulier dans le domaine de l' «évaluation des conséquences économiques, financières, sociales et environnementales » des projets de loi. Les rapports 2014 et 2015 sont l'occasion pour le Conseil de souligner les «domaines d'insuffisance constatées dans les projets qui lui sont soumis ». Insuffisance dans l'exposé des objectifs de la réforme envisagée, insuffisance dans l'exposé des raisons des choix retenus, insuffisance de l'évaluation des conséquences des mesures proposées sur les démarches administratives et la complexité procédurale, insuffisance de l'évaluation des conséquences du texte sur le nouvel ordonnancement juridique qui en résultera, insuffisance du chiffrage des mesures proposées (Rapport CE 2015).

Les causes d'imperfections des études ont été imputées au manque d'expertise, au manque de temps dont souffriraient aussi bien les auteurs des études, que le Conseil d'État, saisi souvent trop tardivement pour exercer un contrôle efficace ou encore les parlementaires pour s'intéresser de près aux dites études, ainsi que par la complaisance que mettraient certains fonctionnaires à aller trop vite dans le sens des choix de leur ministre (Rapport AN 2014).

Pour les trois instruments, les palliatifs aux imperfections notées ont été trouvés dans des renforcements de la portée ou du périmètre de la discipline demandée. En matière de PAP, par des instructions budgétaires de plus en plus complètes quant aux exigences qu'elles mentionnent (Benzérafa et Gibert 2015). Pour l'étude d'impact l'idée du renforcement s'est incarnée dans la demande de suppressions des moyens de contournement de l'obligation de la dite étude (propositions de loi téléguidées par l'exécutif, amendements substantiels, en particulier gouvernementaux apportés aux projets de loi). Pour l'amélioration du contrôle de la qualité du respect de l'obligation il a été également demandée que soit rendue publique la partie de l'avis du Conseil d'État relative à l'étude (AN 2014), (Conseil d'État, 2016). Pour l'étude de partenariat, le renforcement de la discipline peut être vu dans la suppression de

\footnotetext{
${ }^{25}$ Secrétaire d'État chargé des relations avec le Parlement.

${ }^{26}$ Secrétaire d'État à l'enseignement supérieur et à la recherche et précédemment Secrétaire d'État à la réforme de l'État.

${ }^{27}$ Interview donnée au Journal du dimanche du 22 mai 2016 : « Il faut revoir la façon dont on dirige le pays »

${ }^{28}$ En principe, car des fuites peuvent en transparaître dans les médias, et le Gouvernement est toujours libre de rendre publics les avis.
} 
la complexité et de l'urgence comme motifs légitimant le recours au contrat de partenariat (même s'ils sont implicitement réintroduits dans les critères au titre desquels la solution du marché de partenariat peut être considérée comme la meilleure.) La discipline exigée des promoteurs des contrats de PPP, devenus désormais marchés de partenariat, ne ressort pas uniquement du juge administratif qui ne dispose pas d'un pouvoir d'auto-saisine. Un contrôle ex-ante a longtemps été exercé par la Mission d'appui aux PPP (MAPPP), transformée en 2016 en Mission d'appui au financement des infrastructures, sur les critères de recours aux contrats de partenariat, mais également sur la qualité des études concourant au choix de ce mode de passation. De même, les Chambres régionales et territoriales des comptes se sont saisies ex post de nombreux dossiers de PPP dans des domaines aussi différents que les équipements sportifs ou culturels, l'éclairage public, les espaces verts, les transports urbains, les technologies de l'information et de la communication, mettant fréquemment en relief le défaut et les lacunes des études préalables et les écarts en résultant entre les estimations financières initiales et les montants atteints in fine.

Le rapport public annuel 2015 de la Cour des Comptes ${ }^{29}$ en dressant un constat plus que nuancé sur le recours aux contrats de partenariat, mettait en relief le rôle ambigu de la MAPPP sur la période 2004-2014, à la fois expert, contrôleur et promoteur des partenariats public-privé... La Cour pointait précisément la difficulté à faire respecter la discipline managériale que la loi instituait pour les collectivités territoriales. Il est intéressant de relever que cette étude, centrée sur les seuls contrats de partenariats conclus par les collectivités territoriales, considérait que les sommes engagées étaient encore relativement faibles et le risque peu élevé : « (......) le montant cumulé par les investissements réalisés en contrats de partenariat (État et collectivités locales), s'établit pour 2005 à 14,7 Md€. Les 149 contrats signés par les collectivités territoriales sur 10 ans ne représentent que $27 \%$ du total des investissements concernés, soit 4,1 Md€.». La discipline requise semblait ainsi poser une proportionnalité entre le niveau de discipline et les sommes engagées...

\section{Une position prudente du juge qui renforce l'idée d'une percée très relative de la disciplinarisation managériale}

\subsection{La prudence du juge}

Le volet performance des projets annuels de performance (P.A.P) et des rapports annuels de performance (R.A.P) instaurés par la loi organique relative aux lois de finances (LOLF)

Des parlementaires de l'opposition d'alors ont, dès le premier budget établi en mode LOLF (loi de finances pour 2006) et dès le premier recours avant promulgation de la loi de finances devant le Conseil constitutionnel, fait figurer parmi les griefs d'inconstitutionnalité du budget adopté l'insuffisance de l'information qui leur était donnée par les indicateurs de performance. Les 60 députés de l'opposition d'alors signataires du recours énonçaient leurs griefs dans les termes reproduits dans l'encadré 4 .

\footnotetext{
${ }^{29}$ Rapport public de la Cour des Comptes, 2015, les partenariats public-privé des collectivités territoriales : des risques à maîtriser, Paris, $71 \mathrm{p}$.
} 


\section{Encadré 4 : Les indicateurs de performance}

De façon générale, le nombre d'indicateurs de performance non renseignés dans les documents budgétaires transmis au Parlement et détaillant les crédits de chacune des missions est inacceptable et remet en cause la qualité même de l'autorisation parlementaire. Cette défaillance est en contradiction directe avec l'esprit même de la loi organique relative aux lois de finances. Le Gouvernement, qui à de nombreuses reprises a appelé les parlementaires à ne pas se focaliser uniquement sur la progression - souvent négative - des crédits d'une année sur l'autre pour se consacrer à l'étude de la performance et des résultats prive ainsi le Parlement de la capacité d'exercer son contrôle sur l'efficacité des politiques menées et la réalisation, par les responsables de programmes, de leurs objectifs. Comment juger en effet la performance quand les indicateurs qui permettent de l'évaluer ne sont pas construits ou pas renseignés ?Cette absence de renseignement des indicateurs met à mal l'autorisation parlementaire et aura des conséquences dommageables également lors de la discussion du projet de loi de règlement pour l'année 2006. La responsabilisation des gestionnaires publics voulue par la loi organique doit trouver une contrepartie constante dans la nécessité pour eux de s'engager sur des objectifs précis accompagnés des indicateurs permettant d'en mesurer l'achèvement.

À défaut, la qualité du contrôle parlementaire sortirait amoindrie et non renforcée de la réforme.../...

Ce raisonnement faisait appel à l'esprit de la LOLF et à l'idée qu'en contrepartie des libertés budgétaires accrues dont les gestionnaires de l'administration bénéficiaient grâce à la loi organique et grâce en particulier à la fongibilité des crédits qu'elle leur procurait, ces gestionnaires étaient soumis à une redevabilité accrue. Les auteurs du recours critiquaient tout autant le caractère lacunaire de certains indicateurs que l'absence de renseignement d'autres indicateurs. Le Conseil constitutionnel a repris l'idée de l'importance des indicateurs, mais n'a pas fait sienne l'appréciation portée par les députés sur l'ensemble des indicateurs présentés :

Considérant, en l'espèce, qu'il n'est pas établi que les indicateurs de performances associés à la loi de finances pour 2006 soient entachés d'un défaut de sincérité; que, si quelques retards ou déficiences ont pu être constatés et devront être corrigés à l'avenir, ils ne sont, ni par leur nombre, ni par leur ampleur, de nature à remettre en cause la régularité d'ensemble de la procédure législative; que, dès lors, le grief invoqué doit être écarté.

Il y avait, à l'époque de cette décision, deux façons de l'interpréter : la pessimiste revenait à considérer que, compte tenu de l'imperfection réelle des indicateurs présentés, soulignées par ailleurs dans les travaux du C.I.A.P et des commissions parlementaires, le Conseil constitutionnel avait réduit à peu de chose ses exigences en matière de qualité des ambitions affichés par le Gouvernement et de reporting de la performance. L'optimiste consistait à noter que si le raisonnement invoqué par les requérants s'était révélé inefficace en l'espèce, il avait été considéré comme valable par le Conseil constitutionnel car les indicateurs devaient être sincères (restait à définir alors la notion de sincérité d'un indicateur, 
plus délicate encore à cerner que la sincérité d'un budget) et les déficiences ne devaient être ni nombreuses ni flagrantes. Dans cette seconde interprétation, la présentation au Parlement des indicateurs de performance par l'exécutif devenait chose des plus sérieuses.

La lecture des saisines ultérieures du Conseil constitutionnel ne révèle pas que le moyen concernant le budget pour 2006 ait été utilisé à nouveau, ce qui empêche de trancher entre les interprétations pessimiste et optimiste. Or, malgré d'incontestables améliorations touchant à la définition même des indicateurs, à la disparition des indicateurs non alimentés et à la suppression d'indicateurs d'activité, fort éloignés de la traduction opérationnelle des objectifs, les faiblesses fondamentales du volet performance des PAP et des RAP demeurent avec des « présentations stratégiques » qui le sont peu, des objectifs souvent très vagues et des liens entre objectifs et indicateurs par voie de conséquence très discutables (Benzérafa et Gibert, 2015). Il était donc toujours possible de soutenir qu'en de maints endroits le Parlement ne pouvait « se prononcer en connaissance de cause ».

En ce qui concerne la comptabilité d'analyse des coûts, le problème est particulièrement aigu chaque fois que les dépenses de personnels ont été centralisées dans une action au sein d'un programme, et plus encore dans un programme au sein d'une mission. Le chiffrage des seuls crédits directs d'une action ou d'un programme peut donc être très peu représentatif de la réalité des ressources à la disposition de l'action ou du programme. Pour une bonne information du Parlement quant à l'usage prévisionnel des crédits, une ventilation des dépenses indirectes par rapport à une action était donc nécessaire et ce fut-là la première fonction de la CAC. L'enjeu s'est révélé de taille puisque l'on a pu constater que le ratio dépenses complètes (après réception des dépenses indirectes) sur dépenses directes pouvait varier entre des chiffres peu supérieurs à un et des chiffres de l'ordre de la trentaine (Gibert, 2011). Au niveau du constat (loi de règlement et RAP qui lui sont associés), à ce problème s'ajoute la nécessité de doubler l'information sur l'exécution budgétaire (en termes de dépenses et de recettes) par une information en termes de charges et de produits, raisonnement qui est celui de la comptabilité générale de l'État. La présentation ex post doit donc faire passer de la notion de dépenses à celle de charges, et ainsi enregistrer les charges non décaissables (dotation aux amortissements en particulier), tenir compte des variations de stock éventuelles, exclure les dépenses d'investissement et tenir compte des variations des comptes tiers et des charges restant à payer.

La mise en œuvre de ces principes a été effectuée d'une façon que l'on peut qualifier de simple et modeste (Gibert, 2011). La conséquence en est que les déversements effectués peuvent être facilement critiqués en raison des clés utilisées et de la volonté de ne pas opérer de déversements croisés, en raison également du caractère embryonnaire des amortissements opérés et des éventuelles lacunes des comptes de stocks.

Dans le recours exercé contre le premier budget voté en mode LOLF, les auteurs du recours avaient intégré parmi les motifs invoqués à l'appui de leur demande d'annulation le caractère trompeur des dépenses affectées à certaines des politiques en particulier pour la mission «Écologie et développement durable ». Dans son mémoire en défense, le Gouvernement évoquait l'existence de ventilation d'une politique à une autre figurant dans les projets annuels de performance qui permettait selon lui de pallier les inévitables biais pouvant survenir dans la prise en compte des seules dépenses directes. Le Conseil constitutionnel ne s'est pas réellement aventuré sur ce terrain se bornant à poser que «Les choix opérés par le Gouvernement pour déterminer l'architecture des missions (comme d'ailleurs 
celle des programmes et la définition des actions) ou pour rattacher une mission à un ou plusieurs ministères comportent une part incontournable de subjectivité et d'opportunité. Ne pourrait donc être sanctionnée qu'une erreur manifeste d'appréciation dont l'ampleur remettrait en cause l'esprit de la LOLF et la sincérité même de la loi de finances. Tel n'est pas le cas en l'espèce. » (Conseil constitutionnel, 2005). Depuis lors, la question du caractère sincère et donc significatif des coûts affichés n'a pas été abordée, mais il semble clair que le Conseil constitutionnel a tranché en faveur d'un contrôle minimal de sa part en le faisant rentrer dans la sanction éventuelle de la traditionnelle erreur manifeste d'appréciation.

\section{L'étude d'impact ou quand la vérification du respect de la séparation des pouvoirs se substitue au principe de la rationalité de la décision}

Le législateur organique n'a pas, quand on lit l'article 8 de la loi de 2009, lésiné sur les contraintes imposées au pouvoir. Le profane est donc très surpris à la lecture des études d'impact (toutes publiques et disponibles sur le site des assemblées parlementaires) par la légèreté et le caractère lacunaire des documents produits.

Le juge constitutionnel a pourtant compétence pour faire respecter les dispositions de la loi organique précitée, puisqu'aux termes de l'article 39 de la Constitution : «Les projets de loi ne peuvent être inscrits à l'ordre du jour si la Conférence des présidents de la première assemblée saisie constate que les règles fixées par la loi organique sont méconnues. En cas de désaccord entre la Conférence des présidents et le Gouvernement, le président de l'assemblée intéressée ou le Premier ministre peut saisir le Conseil constitutionnel qui statue dans un délai de huit jours » et que de surcroît les parlementaires (60 sénateurs ou 60 députés au moins ont toujours la possibilité de saisir le Conseil constitutionnel avant promulgation de la loi pour en faire censurer les dispositions qu'ils jugent inconstitutionnelles ou contraires aux lois organiques (Article 61 de la constitution). La conjonction de ces deux possibilités n'a cependant pas, jusqu'à présent, favorisé la qualité des études d'impact au regard des exigences de la loi organique.

La conférence des présidents de la première assemblée, saisie du projet de loi, reste liée au phénomène majoritaire. En effet, si les membres - tous ex officio- de la conférence sont d'origine politique variée, le nombre de voix dont dispose chaque groupe politique au sein de cette conférence est de facto fonction de l'importance numérique des groupes politiques dans l'assemblée considérée. Un conflit entre la conférence des présidents et le Gouvernement sur la «méconnaissance des conditions de présentation fixées par la loi organique » est donc un conflit entre le Gouvernement et sa majorité, du moins s'agissant de l'Assemblée nationale. La probabilité que ce conflit naisse des seules conditions de présentation, et en particulier de l'étude d'impact est relativement faible. La première et, jusqu'à ce jour, seule saisine du Conseil constitutionnel avant vote de la loi, s'est produite en 2014 seulement (Combrade, 2014) ${ }^{30}$, et a été due à la position du Sénat à l'égard de l'étude d'impact accompagnant le projet de loi relatif à la délimitation des régions, aux élections régionales et départementales et modifiant le calendrier électoral. Les opposants à un projet de loi n'ont donc, très généralement, comme solution que de déférer au Conseil constitutionnel la loi adoptée par le Parlement avant promulgation. De fait, cette solution a été maintes fois utilisée, 14 fois depuis la mise en place de l'étude d'impact en 2009 jusqu'en mai 2014 (Huttier, 2015).

\footnotetext{
${ }^{30}$ Décision n² 2014-12 FNR du 1 1er juillet 2014.
} 
Dès l'examen de la constitutionnalité de la loi organique ${ }^{31}$, le Conseil révélait qu'il apprécierait de façon souple le respect des dispositions de l'article 8 en formulant deux réserves, l'une pour préciser que l'exigence de procéder à une étude correspondant à chacune des rubriques énumérées par cet article ne s'imposait que pour celles de ces rubriques qui apparaissaient pertinentes au regard de l'objet de la loi, l'autre pour indiquer que « dans le cas où un projet de loi serait déposé sans être accompagné d'une étude d'impact satisfaisant en totalité ou en partie aux prescriptions de l'article 8, il apprécierait le respect de ces dispositions au regard des exigences de la continuité de la vie de la Nation ». Si la première réserve d'un point de vue managérial ne pose aucun problème, la seconde avançait que la raison d'État doit l'emporter sur la rationalité managériale, ce dont on pouvait se douter dans la ligne constante tracée depuis longtemps par le juge administratif, soulignant souvent le caractère second de la rationalité managériale dans la conduite des affaires publiques.

De surcroît le conseil Constitutionnel a durci sa jurisprudence en ce qui concerne l'acceptabilité d'une requête d'annulation au titre d'une insuffisance de l'étude d'impact lorsqu'il est saisi en vertu de l'article 61 de la constitution. Alors que jusqu'en 2015 il avait, à plusieurs reprises accepté de contrôler l'étude d'impact même quand la conférence des présidents de la première assemblée n'avait pas été saisie de la question de la qualité de celle-ci, il conditionne désormais (Décision 2015-718 DC du 13 août 2015) son contrôle à cette saisine ${ }^{32}$. Sans enlever aux membres de l'opposition parlementaire la possibilité de voir leurs griefs en la matière examinés par le Conseil constitutionnel cela la rend pour eux plus difficile à exercer.

En tout état de cause jamais jusqu'ici le Conseil constitutionnel n'a censuré tout ou partie d'un texte de loi au motif d'une insuffisance de l'étude d'impact déposée par le gouvernement. Jamais non plus il n'a explicité les critères sur lesquels il se fondait pour conclure au caractère admissible de l'étude d'impact au regard des exigences de la loi organique tempérées par ses deux réserves interprétatives.

\section{Les marchés de partenariat ${ }^{33}$, ou l'approche opportuniste du management par le juge}

L'évaluation préalable, validée dès 2004 par le législateur et réinscrite au cœur de l'ordonnance du 23 juillet 2015, est désormais considérée comme une formalité substantielle engageant la licéité des opérations réalisées au titre des marchés de partenariat. Cette évaluation préalable, par les méthodes à mobiliser, notamment par l'approche en coût global et le recours à l'analyse de risques, suggère une maturité managériale de la personne publique, et évoque sa capacité à réaliser des valorisations rationnelles, et pour le moins à s'assurer de la soutenabilité budgétaire, condition de la rigueur des projets et... de la discipline de leurs promoteurs politiques ${ }^{34}$.

\footnotetext{
${ }^{31}$ Décision n²009-579 DC du 9 avril 2009.

32 À plusieurs reprises, le Gouvernement dans ses observations avait fait valoir auprès du Conseil constitutionnel une position totalement restrictive, par exemple «si la conférence des présidents accepte l'inscription du texte à l'ordre du jour, le caractère suffisamment complet de l'étude d'impact ne peut plus être contesté. », loi relative à l'élection des conseillers départementaux, des conseillers municipaux et des conseillers communautaires, et modifiant le calendrier électoral (Décision n 2013-667 DC du 16 mai 2013). ${ }^{33}$ Les marchés de partenariat ont remplacé les contrats de Partenariat Public-Privé (PPP) à la faveur de l'ordonnance du 23 juillet 2015 et du décret n²016-360 du 25 mars 2016.

${ }^{34}$ «Pour les marchés de partenariat, l'acheteur réalise, avant le lancement de la procédure de passation, une évaluation ayant pour objet de comparer les différents modes envisageables de réalisation du projet. Cette évaluation comporte une analyse en coût complet et tout élément permettant d'éclairer l'acheteur dans le choix du mode de réalisation de ce projet. » (Article 40 de l'ordonnance du 23 juillet 2015).
} 
Cette conversion du juge constitutionnel à une nécessaire approche managériale des marchés de partenariat - guidant plus de dix années plus tard la rédaction des textes actuels - , est-elle consacrée dans la réalité ? Le juge administratif, garant de la licéité de l'action et non plus seulement de la conformité des intentions, prend-il au mot la loi? Censure-t-il, lorsqu'il en est saisi, les projets de contrats, et bientôt de marchés de partenariat, pour défaut d'évaluation préalable, ou faiblesse dans la méthode de comparaison de ses avantages et de ses inconvénients avec d'autres modes de dévolution?

Depuis la promulgation de l'ordonnance du 17 juin 2004, première consécration des partenariats, 558 projets de contrats, puis de marchés de partenariat, ont fait l'objet d'un avis d'appel à la concurrence, 150 ont été réalisés ou sont en cours de réalisation. Un certain nombre de projets de contrats de partenariat ont fait l'objet de recours contentieux, formés dans la plupart des cas par des associations créées pour en contester le bien-fondé, parfois par des élus locaux d'opposition, et le plus souvent, avant l'entrée en vigueur des nouvelles dispositions, par les syndicats du second œuvre qui voyaient là une restriction à l'accès des PME à ces marchés globaux. Le décret du 25 mars 2016 a anticipé ce dernier reproche en faisant obligation aux administrations d'imposer à leurs partenaires le recours aux $\mathrm{PME}^{35}$.

$\mathrm{Au}$ total, nombre de ces recours reposent sur la problématique classique de l'atteinte aux règles de transparence et de publicité, d'autres malheureusement plus rares, sont fondés sur des considérations de fond tenant à l'absence des critères objectifs de recours aux contrats de partenariat (urgence, complexité), ou au défaut de l'évaluation financière et technique préalable.

L'analyse comporte ici nécessairement les limites que les requérants fixent à leurs propres recours par leur nombre, par les moyens exposés, par leurs finalités mêmes.

Nous choisirons donc, parmi les recours présentés récemment devant le Conseil d'État, deux cas exemplatifs volontairement très différents : le recours sur le projet de réalisation de la Cité de l'Océan et $d u$ Surf à Biarritz ${ }^{36}$, et le recours sur le projet de construction du nouveau Palais de justice de Paris ${ }^{37}$.

Le premier projet est un projet territorial important à l'échelle de la ville de Biarritz mais réduit au niveau national avec un coût global estimé à près de 41 millions $€$ in fine. Le montage en $\mathrm{CP}$ a été contesté dès son origine par un élu d'opposition au Maire de la ville. Par sa décision du 30 juillet 2014, le Conseil d'État a annulé la délibération ${ }^{38}$ autorisant la signature du contrat de partenariat prenant à la lettre les exigences légales liées à la qualité de l'étude préalable comme condition substantielle du contrat de partenariat :

«Le respect des conditions posées par la loi s'apprécie au vu de la qualité de l'évaluation

\footnotetext{
${ }^{35}$ Article 163 du décret n 2016-360 du 25 mars 2016 : « La part minimale que le titulaire s'engage à confier, directement ou indirectement, à des petites et moyennes entreprises ou à des artisans (.../...) est fixée à $10 \%$ du montant prévisionnel du contrat hors coût de financement, sauf lorsque le tissu économique dans le secteur concerné ne le permet pas. »

${ }^{36}$ Conseil d'État, 7e ss. sect., 30 juillet 2014, req. 363007, Biarritz Océan.

${ }^{37}$ CAA de Paris, 3 avril 2014, Ass. La justice dans la cité, n¹3PA02769, N-766, N-770, confirmé par CE, 15 octobre, req. $n^{\circ} 380918$

${ }^{38}$ La théorie jurisprudentielle de l'acte détachable interdit, sur le seul fondement du recours pour excès de pouvoir, de solliciter l'annulation du contrat lui-même. C'est bien l'annulation de la délibération qui est ici en jeu et non pas celle du contrat. Une autre solution jurisprudentielle s'est dégagée avec le recours dit Tropic (CE. Ass., 16 juillet 2007, req. $n^{\circ} 292545$ ), en contestation de validité qui permet d'attaquer directement en annulation les clauses du contrat.
} 
préalable qui a donné lieu à la délibération. (.......) La seule invocation de la complexité des procédés techniques à mettre en œeuvre ne peut suffire à justifier légalement le recours au contrat de partenariat.»

Si l'évaluation préalable du projet «Biarritz-Océan » (......) faisait apparaître de nombreux éléments de complexité technique, il ne ressort pas des pièces du dossier que (... ...) la commune aurait été dans l'impossibilité de définir seule et à l'avance les moyens techniques pouvant répondre à ses besoins ou d'établir le montage financier ou juridique du projet. »

Le respect de la discipline managériale, qui découle ici de l'exigence d'une évaluation préalable du projet, est rappelé sans détour ni timidité au politique. Sans doute devonsnous, pour éviter un procès en naïveté, considérer que cette solution offre au politique, et en l'occurrence à la nouvelle municipalité, l'heureuse opportunité de sortir à bon compte d'un contrat de partenariat qui n'est plus considéré aujourd'hui comme la solution la plus pertinente ${ }^{39}$. Un certain opportunisme du juge administratif (Loschak, 1972) peut avoir partiellement dicté, dans les limites de son contrôle, la solution. Il n'en reste pas moins, que les moyens retenus sont ici très clairement orientés vers la qualité et les méthodes mobilisées par l'évaluation préalable, outil managérial de discipline du politique.

Le second exemple est bien différent quant à son ampleur et ses implications politiques à l'échelle nationale. Il s'agit du projet de transfert du Palais de justice de Paris sur la ZAC de Clichy-Batignolles, promise en son temps à la construction de la cité olympique dans le cadre de la candidature de Paris aux JO 2012. Le projet, confié à l'Établissement public du Palais de justice de Paris (EPPJP), prévoit la conception, la construction, le financement, l'entretien et la maintenance du futur Palais de justice de Paris. Le projet est conclu pour une durée de 27 ans, et un montant global actualisé évalué in fine à 2,4 milliards $€$. L'association «La Justice dans la Cité », ayant pour objet le maintien du TGI de Paris dans l'Ile de la Cité, a formé un recours devant le tribunal administratif (TA), puis devant la Cours administrative d'appel (CAA) de Paris. C'est l'arrêt de la CAA de Paris que le Conseil d'État a confirmé en juillet 2014.

Certains éléments figurant dans l'arrêt de la CAA de Paris peuvent surprendre les observateurs :

- L'approbation de l'évaluation préalable du ministre garde des Sceaux en 2010 était requise avant de définir le périmètre géographique exact du projet par l'EPPJP? Peu importe qu'elle ait été tacite, répond la CAA. «Si les actes administratifs doivent être pris selon les formes et procédures prévues par les lois et règlements, un vice affectant le déroulement d'une procédure administrative préalable n'est pas de nature à entacher d'illégalité la décision prise (......)»

- Les comités techniques paritaires (CTP, à l'époque, aujourd'hui CT), aux termes du statut général de la fonction publique, sont saisis des questions touchant aux problèmes généraux d'organisation, et aux conditions de fonctionnement des administrations. Mais dans le cas d'espèce, peu importe que le CTP du ministère de la justice n'ait pas été saisi

\footnotetext{
${ }^{39}$ La Chambre régionale des Comptes (CRC) avait livré, par son rapport du 26 janvier 2010, sa position sur ce projet, n'épargnant en réalité ni les décideurs, ni les promoteurs ni leur conseiller, la SCET. La MAPPP (Mission d'Appui aux partenariats public-privé) n'échappa pas plus à la critique, la CRC rappelant l'avis favorable émis par la MAPPP (MINEFI) sur le projet.
} 
du projet, répond la CAA de Paris : "La création, dans le ressort de la Cour d'appel de Paris, d'un nouveau Palais de justice rassemblant toutes les juridictions du ressort, ne constitue pas une question générale d'organisation ou de fonctionnement (.......et n'avait donc pas à être soumise au CTP du ministère de la justice. »

- Le recours à un contrat de partenariat doit être justifié par des conditions particulières d'urgence? La dispersion des services du TGI de Paris ne permet pas un fonctionnement normal de la juridiction, répond la CAA de Paris : "Le recours au contrat de partenariat se trouve en l'espèce justifié par l'urgence qui s'attache à la nécessité de mettre fin à une situation particulièrement grave et préjudiciable à l'intérêt général affectant le bon fonctionnement de la justice à Paris...»

La licéité de la procédure fut confirmée par le Conseil d'État, qui ne trouva pas plus à redire que la CAA de Paris, à la conclusion d'un accord dit autonome signé entre l'État, le groupement privé et les établissements financiers, et prévoyant une cession de créance irrévocable à la hauteur du montant in fine. Cet accord autonome fixait les modalités d'indemnisation par l'État de son partenaire privé en cas d'annulation, de résolution, de déclaration de nullité ou de résiliation du contrat de partenariat ou de l'un de ses actes détachables...

«Selon que vous serez puissant ou misérable...». Sans doute, la perspective d'une indemnisation délétère du groupement privé par l'État a-t-elle rendu à la CAA sa puissance créative. Épargner le contribuable national, reste un mobile louable et... d'intérêt général, en quelque sorte. Mais, pour le dessein qui nous occupe ici, la managérialisation du droit en sort considérablement affaiblie.

Ces deux décisions, prises dans un domaine commun et à peu de distance, évoquent une approche des pratiques de bonne gestion et de leur traduction législative à très faible écho, et différentes selon les cas. Le juge administratif semble bien plus attentif à l'effectivité de la règle managériale lorsqu'il s'agit des collectivités territoriales. Le dispositif apparaît moins serré lorsqu'il s'agit de projets par essence, nous ne le contestons pas, très politiques et à très forts enjeux financiers et fonctionnels. Faut-il dans ces conditions inverser notre proposition : N'est-ce pas plutôt le juge qui est discipliné, en termes de management, par le politique?

Malgré (à cause...) des montants exceptionnels en jeu (2,4 milliards pour le Palais de justice, 4,2 milliards quant au siège du ministère de la Défense, réalisé également en marché de partenariat ${ }^{40}$ ), les exigences d'évaluation de l'ordonnance du 17 juin 2004, et désormais de l'ordonnance du 23 juillet 2015, ne sont pas à la hauteur. Tout se passe comme si, inhibé par les enjeux nationaux, le juge n'imaginait pas pouvoir imposer au politique le respect de la rationalité managériale inscrite dans la loi. Ces pratiques gestionnaires de bon usage des deniers ne sont pourtant pas des Soft Law par nature, mais elles le deviennent par destination.

La loi $\mathrm{n}^{\circ}$ 2010-1645 du 28 décembre 2010 de programmation des finances publiques (2011-2014) dans un premier temps, l'ordonnance du 23 juillet 2015 relative aux marchés publics dans un second temps, viennent considérablement limiter le recours aux marchés de partenariat pour les administrations publiques centrales (APUC) autres que les ministères

\footnotetext{
${ }^{40}$ S'agissant de la localisation de ce projet, la Cour des comptes indique dans ses conclusions que «le site de Balard a été choisi sans qu'une étude comparative approfondie ait été menée au préalable ». Sur le plan financier, la Cour des comptes se fait plus sévère encore : « les études préalables menées succinctement par le ministère de la Défense ont fait apparaître un léger avantage financier en faveur du PPP. Mais cet avantage n'est étayé que par des hypothèses aléatoires... ».
} 
eux-mêmes. Ainsi, les établissements publics de santé, les EPST (ex CNRS, INSERM, INRA, INRIA...), les Agence régionales de Santé, les Universités, les musées et théâtre nationaux... ne peuvent plus recourir directement aux marchés de partenariat. Il leur est néanmoins désormais possible d'y recourir indirectement par la médiation de leur ministère de tutelle à la condition que ce dernier soit chargé de l'évaluation préalable et que la soutenabilité budgétaire et financière du projet soit garantie. Faut-il voir dans cette limitation le ferment d'une autodiscipline managériale, ou plutôt celui d'une mise sous tutelle de la stratégie des opérateurs?

\subsection{Une discipline assez peu productive ou l'introduction de managérialismes dans le langage politico-administratif?}

La revue de la jurisprudence relative aux trois dispositifs examinés peut déboucher sur un tableau synoptique confrontant les instruments de gestion requis par la législation et la nature du contrôle exercé par le juge compétent. Suivant la distinction rapportée par Aggeri et Labatut (2010), et en dépit du flou sémantique observable dans la littérature, on considérera que "l'instrument est le produit d'une opération de pensée d'ordre supérieur et comporte "une dimension politique, implicite ou explicite, susceptible d'être révélée dans le cadre d'actions organisées et finalisées alors que l'outil apparaît essentiellement comme un concept technique »(page 9). On a posé ici que les trois dispositifs examinés s'appuient sur des instruments qui, eux-mêmes, font explicitement référence à des outils. Dans les deux premiers cas de figure les instruments doivent être mobilisés pour des objets auxquels ils ne font que concourir : la loi de finance initiale ou la loi de règlement pour les PAP et les RAP, les conditions fixées pour « la présentation des projets de loi déposés devant l'Assemblée nationale ou le Sénat » en ce qui concerne l'étude d'impact. ${ }^{41}$ En ce qui concerne le contrat de partenariat, il y a identité entre l'instrument et l'objet visé. Tant pour les PAP et RAP que pour les études d'impact, il apparaît que le juge s'en tient à un contrôle minimum; il en résulte qu'en règle générale, l'exigence par le juge du respect de la discipline attendue du pouvoir est relativement peu contraignante. Un effet pervers apparaît même, attisé par la conjonction entre l'effort de discipline demandé à l'exécutif et le mode de contrôle du juge : ainsi, seuls les objectifs explicites du projet de loi devraient entrer dans le périmètre de l'étude d'impact ${ }^{42}$, si bien que la sagesse commanderait à être le plus prudent possible en évitant de mettre en relief un trop grand nombre d'objectifs.

La saisine du juge se cantonne pour les PAP-RAP, ainsi que pour l'étude d'impact à la conformité du contenu des instruments-outils à la loi organique les régissant. Dans le cadre du marché de partenariat, s'est ajouté à ce problème récurrent de conformité, celui de la constitutionnalité de l'ordonnance instaurant le contrat de partenariat et de sa loi modificative. Il ressort du tableau ci-dessous qu'en règle générale, l'exigence par le juge du respect

\footnotetext{
${ }^{41}$ Un seul autre instrument est en fait mobilisé à côté de l'étude d'impact : l'exposé des motifs.

42 Décision n²014-12 FNR du 1er juillet 2014, «II ne saurait en particulier être fait grief à cette étude d'impact de ne pas comporter de développements sur l'évolution du nombre des emplois publics dès lors que le Gouvernement ne mentionne pas la modification de ce nombre dans les objectifs poursuivis par ce projet de loi ». Décision citée, à comparer avec les documents constitutifs de l'étude d'impact qui " exposent avec précision (...) l'évaluation des conséquences des dispositions envisagées sur l'emploi public » (cf. loi organique).
} 


\begin{tabular}{|c|c|c|c|c|c|}
\hline 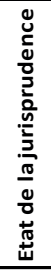 & 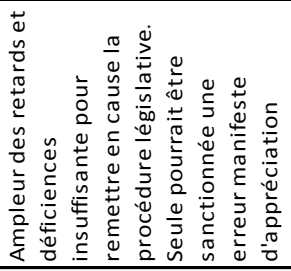 & 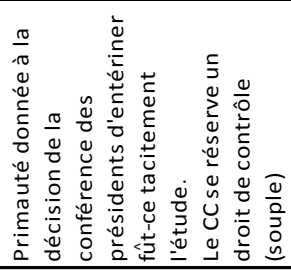 & 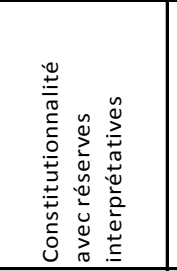 & 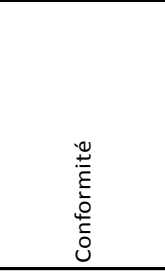 & 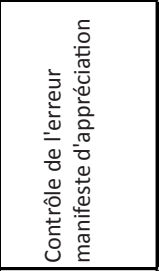 \\
\hline 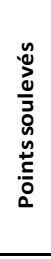 & 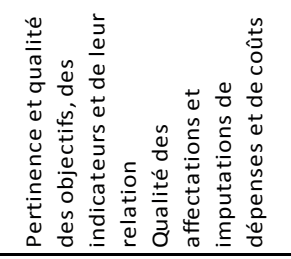 & 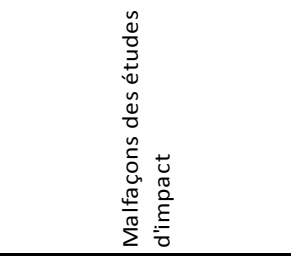 & 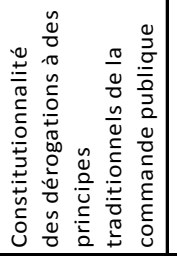 & 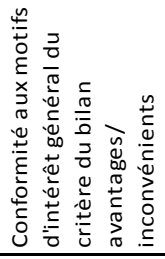 & 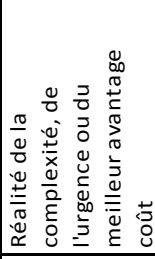 \\
\hline 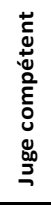 & 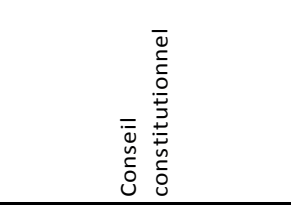 & 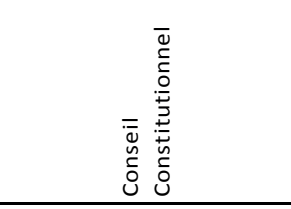 & \multicolumn{2}{|c|}{ 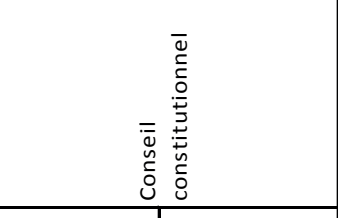 } & 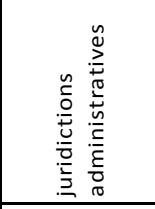 \\
\hline 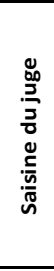 & 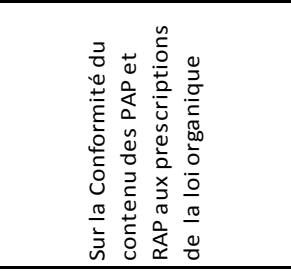 & 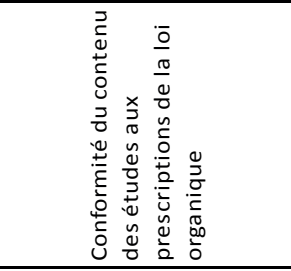 & 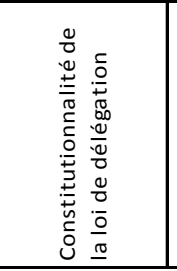 & 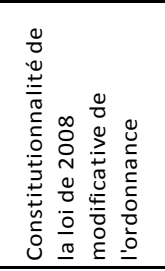 & 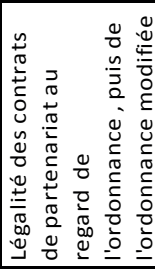 \\
\hline 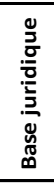 & 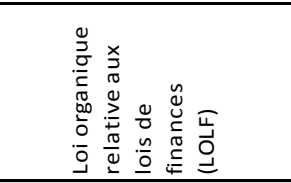 & 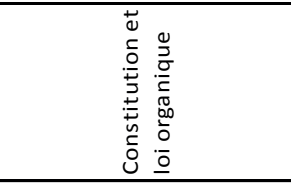 & \multicolumn{3}{|c|}{ 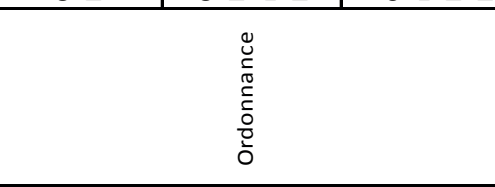 } \\
\hline 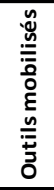 & 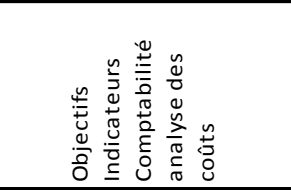 & 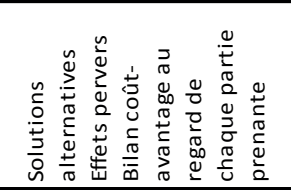 & \multicolumn{3}{|c|}{ 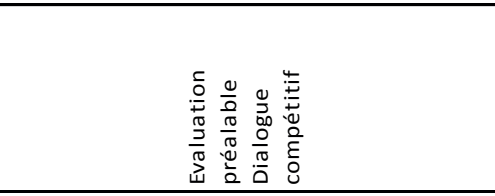 } \\
\hline 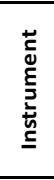 & 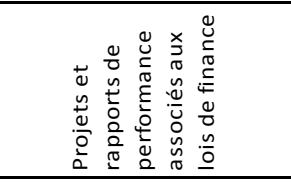 & 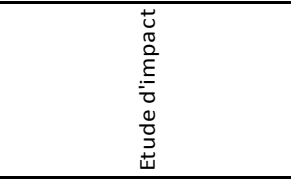 & \multicolumn{3}{|c|}{ 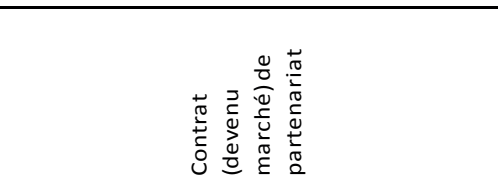 } \\
\hline
\end{tabular}

de la discipline attendue du pouvoir est relativement peu contraignante à l'exception près des contrats de partenariat conclus par des collectivités territoriales. Ce constat interpelle quant à l'apport de l'institutionnalisation des instruments-outils de gestion par rapport à une absence d'institutionnalisation au sens commun du terme.

Lorsqu'une discipline destinée à l'appareil d'État est instaurée par voie réglementaire (Décret, arrêté, voire circulaire), ce qui est le cas à l'heure actuelle des études d'impact relatives aux projets de décrets, elle peut être considérée aussi bien comme une autodis- 
cipline du pouvoir (ce qu'un décret peut faire un décret peut le défaire) que comme une discipline imposée par le pouvoir à l'appareil administratif, dans ce cas on met l'accent sur l'aide à la décision que constitue la discipline en question. Lorsqu'une discipline est instaurée par le moyen d'une loi organique ou ordinaire le doute est permis. En effet, le législateur est décideur en la matière, mais dans la pratique, c'est bien l'exécutif qui est à l'initiative des mesures proposées ${ }^{43}$. En d'autres termes, l'exécutif est à l'origine d'une discipline qui pourra certes être amendée, y compris dans le sens d'un renforcement, par les parlementaires mais que par construction, il accepte pour lui aussi bien que pour ses successeurs. Si l'on exclut l'hypothèse du masochisme des gouvernants, il reste à déterminer les motivations de ceux-ci : besoin d'effectuer une contribution minimale (tokenism) à un principe qui se développe dans la société (meilleure transparence de la gouvernance, plus sérieuse redevabilité des gouvernants) authentique souci de développer la démocratie fut-ce aux dépens des caractéristiques discrétionnaires du pouvoir, croyance en une sorte de concordantisme (ce qui est bon pour la démocratie est bon pour le pouvoir), gage de rééquilibrage des pouvoirs, donné au législatif par un exécutif affaibli... L'autodiscipline, au moins apparente, peut s'accommoder de multiples explications.

En 1968, la Rationalisation des Choix budgétaires fut lancée par un simple arrêté ministériel du MINEFI conforté deux ans plus tard par un décret créant la commission interministérielle des choix budgétaires. C'est dire que le substrat juridique était à l'époque faible et limité à un règlement émanant du pouvoir exécutif s'imposant à lui-même une discipline interne révocable ad nutum.

Quarante années ans plus tard, la budgétisation par programme est consacrée par une loi organique qui ne le cède dans la hiérarchie des normes qu'à la Constitution; l'obligation d'une évaluation a priori est instaurée pour (presque) tous les projets de lois par une autre loi organique, et l'évaluation préalable pour tous les projets de contrat de partenariat est, quant à elle, rendue obligatoire par voie d'ordonnance. C'est dire qu'au premier abord, des principes de bonne gestion ont été institutionnalisés là où ils étaient précédemment laissés aux initiatives du pouvoir exécutif et de ses services.

Cette institutionnalisation est d'autant plus notable qu'elle reste l'exception s'agissant des instruments de gestion utilisables par l'administration. La parution en 2012 du décret de gestion budgétaire et comptable publique (GBCP) a permis de constater que, par rapport au décret sur la comptabilité publique de 1962 qu'il remplace, la référence à la comptabilité analytique est toujours aussi succincte comme si en un demi-siècle la doctrine de l'État en la matière n'avait pu être établie, en dépit de mises en œuvre localisées de comptabilité de gestion et des réflexions de groupes de travail internes à l'administration.

Avec l'étude d'impact (mais mutatis mutandis on pourrait énoncer la même chose pour les deux autres instruments) s'agit-il de passer d'un pouvoir qui affirme (ce qu'est le bien supposé commun) à un pouvoir qui démontre, et dans ce cas quel mode de « démonstration » est-il admis? Un mode hypothético-déductif serré, ce qui renvoie à des démonstrations mathématiques à partir de prémisses fortes ou un mode fondé sur des preuves (Évidence

\footnotetext{
${ }^{43}$ Si la LOLF est issue d'une proposition de loi déposée par deux parlementaires MM. Lambert et Migaud, c'est sous l'œil très favorable du Premier ministre de l'époque (Lionel Jospin) et de son cabinet.
} 
based $)^{44}$ ce qui renvoie à des analyses statistiques, seulement possibles sous la condition d'une disponibilité effective d'informations pertinentes. S'agit-il plutôt d'obliger le pouvoir à passer d'affirmations très globales pour ne pas dire grossières à des explications plus fines, plus désagrégées?

Dans le premier cas de figure c'est le côté discrétionnaire du pouvoir qui est attaqué ainsi que les croyances qui peuvent être les siennes et/ou le recours à une idéologie et/ou au sens commun comme fondement(s) des politiques publiques. C'est l'approche instrumentale des politiques publiques ${ }^{45}$ qui est au moins implicitement mobilisée par l'accent mis sur le travail à effectuer pour que la recherche des effets ou des conséquences débouche sur une efficacité et une efficience. L'exercice de l'étude d'impact est une véritable contrainte pour le pouvoir.

Dans le second cas de figure, seule une image de cohérence interne est requise du pouvoir. La conception cognitive des politiques publiques (Muller, 2000) n'est pas frontalement mise en cause, le sens, les symboles, les représentations... ont encore leur place, mais au prix d'une mise en scène plus précise que la seule évocation de référentiels (Muller, 2000) érigés en dogmes ${ }^{46}$. On peut penser que la discipline peut aider à légitimer le pouvoir et qu'elle est tout autant, sinon plus, ressource que contrainte.

Bien évidemment, on vient d'opposer deux cas extrêmes, il n'y a nulle raison d'exclure des situations intermédiaires explicables par la diversité des inspirations, les luttes des acteurs lors de la mise en œuvre de la discipline ou la faiblesse de la réflexion préalable à l'instauration de la discipline. En tout état de cause le degré d'exigence réel doit pouvoir informer sur la nature même de la discipline. Comme on l'a suggéré plus haut ce degré d'exigence renvoie au contenu même des textes, à l'application qui en est faite, aux réactions que cette application peut soulever ainsi qu'à la réaction du juge compétent aux recours qui lui sont soumis.

En réalité, les instruments de gestion sont aujourd'hui « convoqués » pour donner une image modernisée et rationalisée des rapports entre l'exécutif et le législatif. La juridisation des instruments en question crée des contraintes pour l'exécutif puisqu'elle l'oblige à évaluer davantage le lien entre objectifs et moyens. Cette injonction de transparence met à mal, au premier abord, l'ambiguïté et le flou considérés comme des caractéristiques essentielles de la gestion publique. Mais, s'inscrivant dans le cadre d'un rapport entre les deux pouvoirs, ces instruments changent de finalité : d'instruments de gestion, à proprement

\footnotetext{
${ }^{44}$ Ce qui paraît être la conception de l'OCDE pour laquelle l'analyse d'impact de la réglementation « is an important element of an evidence-based approach to policy making » http://www.oecd.org/gov/regulatorypolicy/ria.htm. Parmi les 12 principes inclus dans la « Recommandation du Conseil concernant la politique et la gouvernance réglementaire » figure le quatrième « Intégrer l'analyse d'impact de la réglementation (AIR) dès le début du processus visant à formuler des projets de réglementation. Définir clairement les objectifs de politique recherchés, déterminer si l'instrument réglementaire est nécessaire et dans quelles conditions il peut être le plus efficace et le plus efficient pour atteindre ces objectifs. Réfléchir à des moyens autres que la réglementation, et faire ressortir les avantages et les inconvénients des différentes approches analysées pour établir laquelle est la meilleure. » http://www.oecd.org/fr/gov/politiquereglementaire/ Recommendation \% 20with \% 20cover \% 20FR.pdf

${ }^{45}$ Celle-ci est de facto prépondérante dans le guide de légistique rédigé par le Secrétariat général du Gouvernement qui dispose que la «norme unilatérale -loi, décret, arrêté...- doit avant tout avoir pour objet de résoudre un problème clairement identifié, en vue d'atteindre un résultat précisément défini pour lequel des solutions non normatives apparaissent manifestement inappropriées. »

${ }^{46}$ Sur un exemple de référentiel érigé en dogme prohibant une véritable analyse « Le livre n'est pas un bien comme un autre » cf. Gibert, 2014.
} 
parler, ils deviennent des instruments de gouvernance (Hood, 1983; Halpern, Lascoumes, Le Galès, 2014), plus précisément des instruments de plaidoyer d'un pouvoir auprès d'un autre, et le cas échéant de l'opinion publique qui peut aisément y avoir accès. C'est là un mode d'appropriation parmi bien d'autres (Vaujany, 2006).

Cette fonction d'advocacy a naturellement des conséquences sur la nature des documents produits. On peut, par exemple, difficilement attendre d'une étude d'impact qu'elle valorise objectivement les solutions alternatives à celle retenue par le Gouvernement, ou qu'elle insiste sur les effets pervers d'un projet de loi. Il ne pourrait en être autrement que si la rigueur du juge constitutionnel dans l'interprétation de la loi organique était reconnue. Mais cette rigueur est, on l'a vu, improbable, en raison du problème de légitimation permanente auquel se heurte tout juge constitutionnel, ensuite parce que la matière managériale est semée d'embuches pour un juge qui ne saurait se voir reprocher son manque d'expertise pour apprécier le périmètre d'un objectif ou la pertinence d'un indicateur, la réalité d'un effet pervers ou le biais introduit par une analyse hypothético-déductive.

Le repli sur l'erreur manifeste d'appréciation comme critère de censure apparaît donc comme une solution « sage », même si elle entérine de facto l'acceptation d'une interprétation assez formelle des contraintes posées par la loi organique et d'une contribution souvent symbolique aux travaux que celle-ci oblige l'exécutif à faire. Dès lors, la contrainte apparaît bien légère et la logique politique finalement peu gênée. L'étude d'impact pousse seulement le Gouvernement à communiquer sur le fait qu'il ne fait pas n'importe quoi (pas de « garbage can » au sens de Cohen, March et Olsen, 1972), que son action n'est pas motivée par de l'idéologie pure ou le seul désir de donner satisfaction à telle ou telle partie de son électorat, ou à un groupe de pression qu'il courtise ou craint. En d'autres termes, l'étude d'impact est un instrument de légitimation de l'action du Gouvernement qui ré-enchante, par la référence à l'avantage coût, la mythologie de l'intérêt général, s'inscrivant par-là dans la continuité de la construction jurisprudentielle de la théorie du bilan, sans risques excessifs pour lui. ${ }^{47}$

Dans le cas des PAP et des RAP, la (maigre) jurisprudence constitutionnelle n'a pas conduit aux mêmes extrémités, sans doute en raison de l'importance même du dispositif de mise en œuvre de la LOLF et du contrôle interne à l'administration (Cour des comptes, CIAP, direction du budget). Elle a même pu, au même titre que la manière dont les programmes ont été définis, aboutir à une certaine routinisation, une amélioration technique beaucoup plus que stratégique, et une résistance assez forte des documents produits aux changements politiques (Benzérafa, Garcin et Gibert, 2014).

Beaucoup plus que la technologie des outils, ce qui importe pour les acteurs dans les exemples étudiés, c'est l'image de rationalité au service de la performance que ces outils véhiculent. Si le management public est un langage (Laufer et Burlaud, 1979), le langage

\footnotetext{
${ }^{47}$ Pour une vision plus optimiste des choses, dans un environnement juridique différent cf. Des Rosiers 2015 qui s'appuyant sur Mc Loughlin pose que « Davantage de contraintes facilitent l'exercice du pouvoir. Les droits de la personne et les exigences d'imputabilité envers les Gouvernements ne seraient plus une contrainte futile mais bien ce qui leur permet d'aller plus loin. L'existence de contraintes procédurales, démocratiques, ou d'imputabilité légitime l'exercice du pouvoir et le rend plus puissant. Le "potestas» soutient la «potentia ». Cette perspective valorise donc des projets Gouvernementaux ambitieux et présente les chartes ou autres instruments d'expression des droits des personnes non comme des carcans, contraintes ou des espaces à minimiser si on veut agir, mais bien comme des véhicules pour favoriser I'action Gouvernementale en lui octroyant davantage de légitimité. »
} 
politico-administratif sait lui emprunter opportunément des termes à connotation positive tout en leur donnant un sens différent. De là, des malentendus volontaires ou non. Les organisations publiques sont un terrain fertile pour les optimisations euphémisantes ${ }^{48}$ s'ajoutant à la collection des oxymores contemporains... Ainsi, quand le politique prétend effectuer une analyse coût/avantage de l'action publique, il ne s'agit pas d'en identifier les effets désirables ou non, d'en prévoir le volume ni de les valoriser chaque fois que possible, mais bien de légitimer le projet en insistant sur ses avantages, et en gommant les effets indésirables. Quand il convient de présenter des objectifs dans le cadre des lois de finances, et de traduire une absence de choix, des pseudo-objectifs purement triviaux suffisent. Comme le législateur et le juge ont en partage le même langage politico-administratif, l'institutionnalisation des instruments de gestion qui pouvait apparaître comme une tentative de discipliner managérialement le pouvoir, repose en fait sur l'incorporation de «managérialismes », de la même manière que la langue française incorpore de plus en plus d'anglicismes.

Si le managérialisme, qui apparaît le plus souvent comme une expression polémique utilisée pour dénoncer l'arrivée de la rationalité managériale qui menacerait la nature même des objets auxquels on voudrait abusivement l'appliquer, est une expression abondamment utilisée fût-ce dans des acceptions très variées (Tandilashvili, 2016), l'idée de managérialismes est peu mise en avant; pour nous, il s'agit de souligner l'importance de l'utilisation du vocabulaire du management à la place de la sémantique administrative traditionnelle sans que ce changement ait un impact réel sur les comportements ou les façons de penser : baptiser un service centre de responsabilité sans qu'il y ait eu une réflexion sur les marges d'autonomie de ce service et la redevabilité (accountability) qui doit lui être associée est un managérialisme, qualifier de stratégie un document qui ne fait que répertorier les compétences juridiques d'un service est un autre managérialisme ${ }^{49}$. C'est l'existence de nombreux managérialismes qui fait que, nouveaux Don Quichotte des anti-managérialistes croyant s'attaquer à des géants, ils s'attaquent à des moulins à vent.

Cet angle de vue ne saurait conduire à exclure les explications plus traditionnelles (comme celle de l'hypocrisie organisationnelle (Brunsson, 1986), ni à conclure systématiquement à une forme d'insincérité des réformateurs de la gouvernance publique. Elle incite cependant à ne pas surestimer ni l'importance de la managérialisation du droit, ni celle de l'illusion rationalisatrice (Rouban, 1993) à laquelle auraient succombé tant de modernisateurs de la gestion publique depuis un demi-siècle.

\footnotetext{
${ }^{48}$ L'emphase dont est coutumier la rhétorique managériale dans l'administration cache parfois des retournements sémantiques étonnants. Ainsi en est-il de l'optimisation utilisée traditionnellement par les économistes dans le sens mathématique et dénoncée comme ambition irréelle par la théorie des organisations pour qui elle s'efface dans la pratique derrière la recherche de solution satisfaisantes. Elle devient un succédané couramment utilisé dans le public (PAP, collectivités territoriales) à l'amélioration de l'efficience quand celle-ci est considérée comme politiquement ou syndicalement incorrecte. Ironie de l'histoire quand on sait que le succès du terme d'efficience, dans l'administration française a été en partie dû dans les années 1980 à l'évitement du terme, alors honni par les syndicats de fonctionnaires, de productivité!

${ }^{49} \mathrm{Ce}$ qui ne veut pas dire qu'à la longue le vocabulaire ne finira pas par recouvrir une réalité.
} 


\section{Conclusion}

Nous avons examiné les problèmes soulevés par la mise en œuvre de trois prescriptions incarnées par des lois organiques dans deux cas et ordinaire dans le dernier et visant à discipliner le pouvoir en lui prescrivant d'expliciter les objectifs des choix qui sont les siens et la raison des moyens qu'il compte utiliser pour atteindre ces objectifs. Nous avons observé directement (on veut dire par nos propres études) dans le cas de la LOLF, de façon indirecte en analysant les réactions du milieu politique ainsi que du Conseil d'État dans le cas de l'étude d'impact des projets de loi, par analyse d'un petit nombre de cas en ce qui concerne le cas de l'étude préalable aux contrats (devenus marchés) de partenariat que l'observation de ses prescriptions laissait pour le moins à désirer et que l'examen de la jurisprudence du conseil constitutionnel et des juridictions administratives révélait un degré d'exigence pour le moins modéré au regard des textes prescriptifs. Nous avons ainsi illustré l'idée selon laquelle l'institutionnalisation de normes managériales se heurte à la force d'une rationalité politique qui s'accommode mal de la précision des objectifs, de l'explicitation d'une véritable théorie d'action reliant objectifs recherchés et moyens retenus. Nous avons également illustré l'idée que le juge constitutionnel ou administratif sensible aux difficultés de la gouvernance fait preuve pour cela, en ce qui concerne l'État du moins d'une mansuétude par une interprétation minimaliste de son contrôle sur l'application des règles managériales.

\section{Notre travail présente au moins quatre séries de limites.}

La première tient au fait qu'il a été centré sur le seul cas français, on ne peut exclure que dans des pays étrangers où l'idée d'instrumentalité des politiques publiques est moins concurrencée qu'en France par les approches cognitives, où l'accent sur les conséquences des politiques publiques est prédominant, où la vision du droit paraît plus instrumentale, où la culture évaluative est plus prégnante le degré réel d'exigence et de respect des normes managériales soit plus élevé. C'est ce que semblent penser les juristes du Conseil d'État quand ils notent « la conception française... s'attache tant à la clarté de la norme qu'à son caractère lisible et compréhensible par le plus grand nombre. Dans d'autres États comme aux Pays Bas ou au Royaume-Uni, l'accent est davantage mis sur les effets de la norme sur l'individu ou sur l'activité économique et la charge administrative qu'elle représente... Sans renoncer à sa traduction d'excellence juridique notre pays doit lui aussi partager cette ambition » (CE 2016 b).

La deuxième limite tient aux méthodes utilisées pour l'appréciation de l'étude d'impact des projets de loi et de l'étude préalable au contrat-marché de partenariat. On ne dispose pas d'une revue systématique des études réalisées dans l'un et l'autre cas, dès lors les limites énoncées à l'égard des études effectuées sont fondées sur des exemples illustratifs mais pas forcément statistiquement significatifs ou sur des opinions de personnes ou d'autorités qui peuvent avoir d'importants biais de jugement (utilisation consciente ou non de critères à proprement parler politiques ou bien corporatistes...). Des études statistiques sur échantillonnage et à partir de l'objectivisation de critères au regard des prescriptions législatives seraient les bienvenues dans les deux domaines mentionnés.

Une troisième limite pourrait tenir à la relative nouveauté de l'obligation instaurée au moins en matière d'étude d'impact et d'étude préalable. L'explication d'imperfections et 
de lacunes qui seraient dues à l'inexpérience et qui pourrait s'atténuer, avec le temps, en fonction d'une capitalisation de l'apprentissage est en effet, une hypothèse concurrente mais partiellement compatible avec celle que nous avons privilégiée d'opposition entre rationalité politique et rationalité managériale. La validation de cette hypothèse suppose elle aussi des travaux ultérieurs. Nous noterons cependant que l'examen de l'évolution du respect des prescriptions de la LOLF dans le domaine de la présentation des objectifs et d'indicateurs sur une période déjà un peu longue de 9 ans s'est traduite davantage par une précision du genre que constitue le volet performance des PAP qui fait diminuer l'hétérogénéité de présentation en la matière des PAP que par un réel effet d'apprentissage. (Benzérafa, Garcin et Gibert, 2016)

La quatrième limite tient au fait que la faible effectivité de la législation quant à l'introduction récente d'instruments de gestion, ne saurait faire conclure à l'inanité des tentatives de « modernisation de la gestion publique ». À côté en effet de l'institutionnalisation de certains instruments de gestion, se poursuit le déploiement d'instruments non portés véritablement par le droit : Indicateurs, tableaux de bord, systèmes de contrôle, continuent à se développer au sein des ministères, des établissements publics ou des collectivités territoriales de la même manière que se développent des évaluations essentiellement ex post. Certes, l'ensemble est moins visible de l'extérieur que ne le sont les instruments «juridicisés ». Par nature, cet ensemble est plus en prise avec la gestion quotidienne, et les problèmes qu'elle pose aux managers publics. Simplement, aucun instrument du management public n'est assuré de sa pérennité. L'avenir des instruments dépend essentiellement des modes; il est tributaire des changements de responsables, de leur mode personnel de gestion, et des aléas politique. Si la discipline subie est d'une portée faible, l'autodiscipline peut être d'une pérennité limitée...

\section{Bibliographie}

Aggeri F. et Labatut J., 2010. La gestion au prisme de ses instruments. Une analyse généalogique des approches théoriques fondées sur les instruments de gestion. Finance Contrôle Stratégie 13 (3), 5-37.

Benzérafa M., Garcin L., Gibert P. et Gueugnon J.-F., 2011. Le management par objectifs met-il fin à l'ambiguïté dans la gestion publique? Politiques et management public 28 (4), 353-389.

Benzérafa M., Gibert P., 2015. Dynamique des indicateurs de reporting externe : le cas des indicateurs des projets et rapport annuels de performance annexés aux lois de finances. Revue française d'administration publique $155,763-778$.

Benzérafa M., Garcin L. et Gibert P., 2016. Le volet performance de la LOLF - Standardisation et résilience d'un genre entre rationalité politique et rationalité de gestion. Revue française de gestion 260,11-31.

Bezes P., 2002. Gouverner l'administration. Une sociologie des politiques de la réforme administrative en France (1962-1997). Thèse de doctorat en science politique, Institut d'études politiques de Paris.

Bezes P., 2005. L'État et les savoirs managériaux : essor et développement de la gestion publique en France In F. Lacasse et P.-E. Verrier (dirs.), Trente ans de réforme de l'État, Dunod, Paris, 9-40.

Bezes P., 2008. Le tournant néomanagérial de l'administration française In O. Borraz, V. Guiraudon (dirs.), Politiques publiques, tome I, Les Presses de Sciences Po, Paris, 215-254.

Brunetière J.-R., 2006. Les indicateurs de la loi organique relative aux lois de finances (LOLF) : une occasion de débat démocratique? Revue française d'administration publique 117, 95-111.

Brunetière J.-R., 2010. Les objectifs et les indicateurs de la LOLF, quatre ans après... Revue française d'administration publique 135, 477-495. 
Brunsson N., 1986. Organizing for Inconsistencies : on Organizational Conflict, Depression and Hypocrisy as Substitutes for Action. Scandinavian Journal of Management Studies 2-3, 165-185.

Caillosse J., 1989. L'administration française doit-elle s'évader du droit administratif pour relever le défi de l'efficience? Politiques et management public 7 (2), 163-182.

Caillosse J., 2003. Les figures croisées du juriste et du manager dans la politique française de réforme de l'État. Revue française d'administration publique 105-106, 115-134.

Campagnac É., 2009. Contribution à l'analyse des contrats de partenariat public-privé en France et au RoyaumeUni. Revue française d'administration publique 130, 365-382.

Chevalier J., 1993. Management public et droit. Politiques et management public 26 (3), 93-100.

Chevalier J., 1993. La juridicisation des préceptes managériaux. Politiques et management public 11 (4), 111-134.

Chun Y. H. and Rainey H., 2005. Goal Ambiguity in U.S. Federal Agencies. Journal of Public Administration Research and Theory 15 (1), 529-557.

Cohen M. D., March J. and Olsen J., 1972. A Garbage can Model of Organizational Choice. Administrative Science Quarterly 17 (1), 1-25.

Combrade B.-L., 2014. Cinq ans plus tard : première (et dernière ?) application de l'article 39, alinéa 4 de la Constitution. Petites affiches 171, 6-11.

Crête J., 2014. Les surveillants de l'État démocratique. Presses de l'Université de Laval, Laval.

Di Manno T., 1998. Le juge constitutionnel et la technique des décisions interprétatives en France et en Italie. Revue internationale de droit comparée 50 (1), 263-266.

Des Rosiers N., 2015. Pour une Charte de Bonne Gouvernance Publique. Revue québécoise de droit international, Ottawa Faculty of Law Working Paper, $n^{\circ} 2015-24,171-182$.

Dror Y., 1967. Policy Analysts : A new Professional Role in Government Service. Public Administration Review 27 (3), 197-203.

Duran P., 1993. Piloter l'action publique, avec ou sans le droit? Politiques et management public 11 (4), 1-45.

Duran P., 2009. Légitimité, droit et action publique. L'Année sociologique 59 (2), 303-344.

Gibert P., 2011. La comptabilité d'analyse des coûts de la LOLF, petit jeu et grand enjeu In C. Hoarau, Malo J.-L. et Simon C., Comptabilité, contrôle et société, mélanges en l'honneur du professeur Alain Burlaud, Foucher, Paris, 293-306.

Gibert P., 2014. Savoir ou légitimer? Les indicateurs et la théorie d'action dans le maelström des évaluations. Le cas de la loi Lang In Tricou F. et Leeman D. éds, Économie, mathématiques et histoire hommage à Christian Bidard, Presses universitaires de Paris-Ouest, Nanterre, 287-304.

Gibert P., 2015. Quel management public? Économie et management 154, 5-12.

Foucault M., 1975. Surveiller et punir, Naissance de la prison. Gallimard, Paris.

Godard O., 2000. Le principe de précaution, un principe politique d'action. Revue Juridique de l'Environnement 25 (1), 127-144.

Halpern C., Lascoumes P. et Le Galès P. (dir.), 2014. L'instrumentation de l'action publique. Presses de Sciences Po, Paris.

Hood C., 1983, 1986. The Tools of Government. Chatham House Publishers, London.

Hutier S. 2015. Retour sur un moyen récurrent: les malfaçons de l'étude d'impact des projets de loi. Revue française de droit constitutionnel 101, 73-86.

Jan P., 2012. Bloc de constitutionnalité. Jurisclasseur Administratif 11 (1418).

Kaplan R. S. and Norton D. P., 2001. The Strategy-Focused Organization : How Balanced Scorecard Companies Thrive in the new Business Environment. Harvard Business School Press, Boston.

Kelsen H., 1996. Théorie générale des normes. PUF, Paris.

Kirat T. et Marty F., 2007. Les enjeux comptables et budgétaires des PPP. Pouvoirs locaux 74, 59-64.

Le Texier T., 2011. Foucault, le pouvoir et l'entreprise : pour une théorie de la gouvernementalité managériale. Revue de philosophie économique 12, 53-85. 
Le Texier T., 2016. Le Maniement des hommes. Essai sur la rationalité managériale. La Découverte, Paris.

Loschak D., 1972. Le rôle politique du juge administratif. LGDJ, Paris.

Marty F., Trosa S. et Voisin A., 2003. Les partenariats public-privé : Démission ou retour de la Puissance publique? La lettre du management public 45.

Marty F., Trosa S. et Voisin A., 2006. Les partenariats public-privé. La Découverte, Paris.

Muller P., 2000. L'analyse cognitive des politiques publiques : vers une sociologie politique de l'action publique. Revue française de science politique 50 (2), 189-208.

Noreau P., 2000. La norme, le commandement et la loi : le droit comme objet d'analyse interdisciplinaire. Politique et sociétés 19 (2-3), 153-177.

Rose N. and Miller P., 1992. Political Power beyond the State : Problematics of Government. The British Journal of Sociology 43 (2), 173-205.

Rouban L., 1993. L'évaluation, nouvel avatar de la rationalisation administrative ? Les limites de l'import-export institutionnel. Revue française d'administration publique 66, 197-208.

Star S. L. and Griesemer J. R., 1989. Institutional Ecology, "Translations" and Boundary Objects : Amateurs and Professionals in Berkeley's Museum of Vertebrate Zoology, 1907-39. Social Studies of Science 19 (3), $387-420$.

Tandilashvili N., 2016. le managérialisme et l'identité universitaire ; le cas de l'université française. Thèse pour le doctorat en sciences de gestion, Université de paris Ouest Nanterre la Défense.

Troper M., 2014. La séparation des pouvoirs et l'histoire constitutionnelle française. LGDJ, Paris.

De Vaujany F.-X., 2006. Pour une théorie de l'appropriation des outils de gestion : vers un dépassement de l'opposition conception-usage. Management \& Avenir 9, 109-126.

Verrier P.-E., 1985. Les «statues » de la fonction publique : les juristes et leurs prédateurs dans le système administratif. Politiques et management public 3 (3), 1-20.

Vigour C., 2006. Justice: l'introduction d'une rationalité managériale comme euphémisation des enjeux politiques. Droit et société 63-64 (2-3), 425-455.

Wildavsky A., 1969. Rescuing Policy Analysis from PPBS. Public Administration Review 29 (2), 189-202. Version française : Sauver l'analyse de politique du PPBS. Politiques et management public 9 (4), 75-98.

Willmann Ch., 2015. Loi pour la croissance, l'activité et l'égalité des chances économiques... et l'emploi? Droit social $10,767-777$.

\section{Documents parlementaires ou administratifs cités}

Assemblée Nationale, 2009. Rapport $N^{\circ} 1375$ fait au nom de la commission des lois constitutionnelles de la législation et de l'administration générale de la république sur le projet de loi organique $\left(N^{\circ} 1314\right)$ relatif à l'application des articles 34-1, 39 et 44 de la Constitution, par Jean Luc Warsmann.

Assemblée Nationale, 2014. Rapport d'information fait au nom de la mission d'information sur la simplification administrative.

Comité de réflexion et de proposition sur la modernisation et le rééquilibrage des institutions de la Ve République présidé par Édouard Balladur, 2007. Une V République plus démocratique.

Comité interministériel d'audit des programmes (CIAP), Rapports d'activité des: $2^{\mathrm{e}}$ cycle (2004/2005) octobre $2005,3^{\text {e }}$ cycle $(2005 / 2006)$ novembre $2006,4^{\text {e }}(2006 / 2007)$ octobre $2007,7^{\text {e }}$ cycle $(2009 / 2010)$ décembre 2010, 8 e cycle (2010/2011) décembre 2011.

Conseil d'État, 2016. Étude annuelle 2016, simplification et qualité du droit.

Cour des comptes, 2011. La mise en æeuvre de la loi organique relative aux lois de finances (LOLF).

Cour des comptes, 2015. Les partenariats public-privé des collectivités territoriales : des risques à maîtriser.

Mission d'appui à la réalisation des contrats de partenariat, 2009. Guide méthodologique.

Secrétariat général du gouvernement, 2015. Guide de légistique. Version par fiches actualisées. https://www. legifrance.gouv.fr/Droit-francais/Guide-de-legistique. 